ESAIM: COCV 20 (2014) 894-923

DOI: $10.1051 / \mathrm{cocv} / 2014001$
ESAIM: Control, Optimisation and Calculus of Variations

www.esaim-cocv.org

\title{
ON THE RELATION OF DELAY EQUATIONS TO FIRST-ORDER HYPERBOLIC PARTIAL DIFFERENTIAL EQUATIONS
}

\author{
IASSON Karafyllis ${ }^{1}$ AND Miroslav Krstic ${ }^{2}$
}

\begin{abstract}
This paper establishes the equivalence between systems described by a single first-order hyperbolic partial differential equation and systems described by integral delay equations. Systemtheoretic results are provided for both classes of systems (among them converse Lyapunov results). The proposed framework can allow the study of discontinuous solutions for nonlinear systems described by a single first-order hyperbolic partial differential equation under the effect of measurable inputs acting on the boundary and/or on the differential equation. Illustrative examples show that the conversion of a system described by a single first-order hyperbolic partial differential equation to an integral delay system can simplify considerably the stability analysis and the solution of robust feedback stabilization problems.
\end{abstract}

Mathematics Subject Classification. 34K20, 35L04, 35L60, 93D20, 34K05, 93C23.

Received February 5, 2013. Revised July 14, 2013.

Published online June 13, 2014.

\section{INTRODUCTION}

The relation of first-order hyperbolic Partial Differential Equations (PDEs) with delay equations is well known. In many cases, a system of First-Order Hyperbolic PDEs (FOH-PDEs) can be transformed to a system described by Retarded Functional Differential Equations (see $[4,10,11,25,27,28]$ ), On the other hand, the recent works [14-16] have shown that systems of first-order hyperbolic PDEs can be utilized for the stabilization of delay systems.

However, recent works on the control of systems described by FOH-PDEs (see $[1-3,5-7,14,21,23,24,30,31]$ ) have led to the study of non-standard systems of FOH-PDEs with the following features: (i) the boundary conditions are given by functionals of the whole state profile; and (ii) the differential equations involve functionals of the whole state profile and not only point values of the states. It should be noted that for such systems there is no available existence-uniqueness theory similar to the theory of standard FOH-PDEs (see [17, 26]): the researchers have utilized transformation arguments which can guarantee important system-theoretic properties. Moreover, the existence of functionals of the whole state profile in the mathematical description of such systems do not allow the answer to the following question:

\footnotetext{
Keywords and phrases. Integral delay equations, first-order hyperbolic partial differential equations, nonlinear systems.

1 Department of Mathematics, National Technical University of Athens, Zografou Campus, 15780, Athens, Greece. iasonkar@central.ntua.gr

2 Department of Mechanical and Aerospace Engineering, University of California, San Diego, La Jolla, CA 92093-0411, USA. krstic@ucsd.edu
} 
"What class of delay systems can be used for the description of non-standard systems of FOH-PDEs?"

In this work, we answer the above question for a class of systems described by a single FOH-PDE. First, we show that the appropriate class of delay systems is a class which has been rarely studied, i.e., systems of the form:

$$
\begin{aligned}
& \eta(t)=f\left(\eta_{t}, w_{t}\right) \\
& \eta(t) \in \mathbb{R}^{n}, w(t) \in W \subseteq \mathbb{R}^{m}
\end{aligned}
$$

where $W \subseteq \mathbb{R}^{m}$ is a non-empty closed set with $0 \in W, r>0, \eta_{t} \in L^{\infty}\left([-r, 0) ; \mathbb{R}^{n}\right), w_{t} \in L^{\infty}([-r, 0] ; W)$ are defined by $\left(\eta_{t}\right)(s)=\eta(t+s)$ for $s \in[-r, 0),\left(w_{t}\right)(s)=w(t+s)$ for $s \in[-r, 0]$ and $f: L^{\infty}\left([-r, 0) ; \mathbb{R}^{n}\right) \times$ $L^{\infty}([-r, 0] ; W) \rightarrow \mathbb{R}^{n}$ is a mapping. We call the above class of delay systems a system described by Integral Delay Equations (IDEs). Systems of the form (1.1) have been studied in [11,18-20,22]. It should be noted at this point that systems described by IDEs have been utilized for a long time in the stabilization of finitedimensional control systems with input delays (see $[13,15,16]$ ): every predictor feedback is a system described by an IDE, whose input is the state of the finite-dimensional control system. Indeed, a predictor feedback for the finite-dimensional system

$$
\begin{aligned}
& \dot{y}(t)=g(y(t), \eta(t-r)+v(t)) \\
& y(t) \in \mathbb{R}^{k}, \eta(t) \in \mathbb{R}^{n}, v(t) \in \mathbb{R}^{n}
\end{aligned}
$$

where we use $x$ to denote the control input and $v \in L_{\mathrm{loc}}^{\infty}\left(\mathbb{R}_{+} ; \mathbb{R}^{n}\right)$ denotes the control actuator error, results in a static feedback law of the form:

$$
\eta(t)=f\left(\eta_{t}, y(t)+u(t)\right)
$$

where $u \in L_{\text {loc }}^{\infty}\left(\mathbb{R}_{+} ; \mathbb{R}^{n}\right)$ denotes the measurement error. Clearly, the above closed-loop system is the feedback interconnection of an time-invariant system described by ODEs and an integral delay system of the form (1.1). Therefore, the study of systems described by IDEs is important on its own. Important system-theoretic properties for the class of systems described by IDEs are provided in Section 2 of the present paper.

Secondly, we show that a class of systems described by a non-standard single FOH-PDE, namely systems of the form:

$$
\begin{gathered}
\frac{\partial x}{\partial t}(t, z)+c \frac{\partial x}{\partial z}(t, z)=a(p(t), z) x(t, z)+g(z) p(t), \text { for } t>0, z \in(0,1) \\
p_{i}(t)=K_{i}\left(w(t), x_{t}\right), \text { for } t>0, i=1, \ldots, N \text { and } p(t)=\left(p_{1}(t), \ldots, p_{N}(t)\right)^{\prime} \in \mathbb{R}^{N} \\
x(t, 0)=G\left(w(t), x_{t}\right), \text { for } t \geq 0
\end{gathered}
$$

where $N>0$ is an integer, $x(t, z) \in \mathbb{R}, w(t) \in W \subseteq \mathbb{R}^{m}, W \subseteq \mathbb{R}^{m}$ is a closed set, $c>0$ is a constant, $g(z)=\left(g_{1}(z), \ldots, g_{N}(z)\right), G: W \times L^{\infty}((0,1] ; \mathbb{R}) \rightarrow \mathbb{R}, K_{i}: W \times L^{\infty}((0,1] ; \mathbb{R}) \rightarrow \mathbb{R}(i=1, \ldots, N)$ are functionals, $a(p, z), g_{i}(z)(i=1, \ldots, N)$ are sufficiently regular scalar functions and $x_{t}$ is the state profile (i.e., $\left(x_{t}\right)(z)=x(t, z)$ for all $t \geq 0$ and $\left.z \in[0,1]\right)$, are equivalent to systems described by IDEs. The equivalence allows the development of important system-theoretic results for the class of systems described by (1.2), (1.3) and (1.4) (Section 3 of the present paper). The obtained results can allow the study of measurable inputs $w(t) \in W \subseteq \mathbb{R}^{m}$, which is an important feature because in many cases boundary conditions of the form (1.4) result from the implementation of stabilizing feedback laws (see [1-3,5,7,14,23,24]). However, the control action comes together with control actuator errors and measurement errors, which are typically modeled by measurable inputs.

Finally, the obtained results can allow the development of a methodology for the solution of control problems for systems described by a non-standard single FOH-PDE, namely systems of the form (1.2), (1.3), (1.4). The methodology is presented in Section 4 of the present paper by means of three illustrative examples. The examples show many important features of the proposed methodology and a comparison is made with other existing methodologies. 
Notation. Throughout this paper, we adopt the following notation:

$* \mathbb{R}_{+}:=[0,+\infty)$.

* Let $I \subseteq \mathbb{R}$ be an interval and $U \subseteq \mathbb{R}^{m}$ be a set. By $L^{\infty}(I ; U)$ we denote the space of measurable and essentially bounded functions $u(\cdot)$ defined on $I$ and taking values in $U \subseteq \mathbb{R}^{m}$. By $L^{\mu}(I ; U)$, where $\mu \in[1,+\infty)$, we denote the space of measurable functions $u(\cdot)$ defined on $I$ and taking values in $U \subseteq \mathbb{R}^{m}$ for which $\int_{I}|u(s)|^{\mu} \mathrm{d} s<+\infty$. By $L_{\text {loc }}^{\infty}(I ; U)$ we denote the space of measurable and locally essentially bounded functions $u(\cdot)$ defined on $I$ and taking values in $U \subseteq \mathbb{R}^{m}$, i.e., for every compact interval $J \subseteq I$ it holds that $u \in L^{\infty}(J ; U)$. For every $x \in L^{\infty}\left(I ; \mathbb{R}^{n}\right)$, where $I \subseteq \mathbb{R}$ is an interval, we denote by $\|x\|$ the essential supremum of $x$, i.e., $\|x\|=\sup _{s \in I}|x(s)|$. For $x \in L^{\infty}\left(I ; \mathbb{R}^{n}\right)$, where $I \subseteq \mathbb{R}$ is an interval with $[a, b) \subseteq I, a<b$, we define $\|x\|_{[a, b)}=\sup _{a \leq s<b}|x(s)|$.

* For a vector $x \in \mathbb{R}^{n}$, we denote by $x^{\prime}$ its transpose and by $|x|$ its Euclidean norm.

\section{SySTEMS DESCRIBED By INTEGRAL DELAY EQUATIONS}

We consider system (1.1) under the following assumptions:

(H1) There exist non-decreasing functions $a: \mathbb{R}_{+} \rightarrow \mathbb{R}_{+}, M: \mathbb{R}_{+} \rightarrow \mathbb{R}_{+}, N: \mathbb{R}_{+} \rightarrow \mathbb{R}_{+}$such that for every $R>0$ and for every $\eta, y \in L^{\infty}\left([-r, 0) ; \mathbb{R}^{n}\right), w \in L^{\infty}([-r, 0] ; W)$ with $\|\eta\| \leq R,\|y\| \leq R,\|w\| \leq R$ the following inequalities hold:

$$
\begin{gathered}
|f(\eta, w)-f(y, w)| \leq N(R) h \sup _{-h \leq s<0}|\eta(s)-y(s)|+M(R) \sup _{-r \leq s<-h}|\eta(s)-y(s)|, \text { for all } h \in(0, r) \\
|f(\eta, w)| \leq a(R)
\end{gathered}
$$

(H2) For every $\delta>0, \eta \in L^{\infty}\left([-r, \delta) ; \mathbb{R}^{n}\right), w \in L^{\infty}([-r, \delta] ; W)$, the function $z:[-r, \delta) \rightarrow \mathbb{R}^{n}$ defined by $z(t)=\eta(t)$ for $t \in[-r, 0)$ a.e. and $z(t)=f\left(\eta_{t}, w_{t}\right)$ for $t \in[0, \delta)$ a.e. satisfies $z \in L^{\infty}\left([-r, \delta) ; \mathbb{R}^{n}\right)$.

Systems of the form (1.1) can represent integral delay equations of the form:

$$
\eta(t)=\sum_{i=1}^{N} p_{i}\left(\eta\left(t-\tau_{i}\right), w\left(t-\tau_{i}\right)\right)+\int_{0}^{r} q(s, \eta(t-s), w(t-s)) \mathrm{d} s
$$

where $r>0,0<\tau_{1} \leq \tau_{2} \leq \ldots \leq \tau_{N} \leq r$ are constants, $p_{i}: \mathbb{R}^{n} \times W \rightarrow \mathbb{R}^{n}(i=1, \ldots, N)$ are locally Lipschitz mappings, $W \subseteq \mathbb{R}^{m}$ is a non-empty closed set and $q: \mathbb{R} \times \mathbb{R}^{n} \times W \rightarrow \mathbb{R}^{n}$ is a locally Lipschitz mapping. It can be shown that assumptions (H1), (H2) hold for this class of systems. The fact that assumption (H2) holds for this class of systems is a direct consequence of Lemma 1 on page 4 in [8].

The following result shows us that system (1.1) can be regarded as a time-invariant system with inputs, whose state space is $L^{\infty}\left([-r, 0) ; \mathbb{R}^{n}\right)$ and whose input set is $L^{\infty}([-r, 0] ; W)$. System (1.1) satisfies the classical semigroup property, the Boundedness-Implies-Continuation (BIC) property and the property of Lipschitz dependence on the initial conditions (see [12]).

Theorem 2.1. Consider system (1.1) under assumptions (H1), (H2). Then for every $\eta_{0} \in L^{\infty}\left([-r, 0) ; \mathbb{R}^{n}\right), w \in$ $L_{\mathrm{loc}}^{\infty}([-r,+\infty) ; W)$ there exists $t_{\max }=t_{\max }\left(\eta_{0}, w\right) \in(0,+\infty]$ with $t_{\max }>\frac{r}{1+2 r N\left(5 a\left(\max \left(\left\|\eta_{0}\right\|, \sup _{-r \leq s \leq r}|w(s)|\right)\right)\right)}$

and a unique mapping $\eta \in L_{\mathrm{loc}}^{\infty}\left(\left[-r, t_{\max }\right) ; \mathbb{R}^{n}\right)$ satisfying $\eta(t)=f\left(\eta_{t}, w_{t}\right)$ for $t \in\left[0, t_{\max }\right)$ a.e. and $\eta(t)=\eta_{0}(t)$ for $t \in[-r, 0)$ a.e.. Moreover, if $t_{\max }<+\infty$ then $\lim \sup \left\|\eta_{t}\right\|=+\infty$. Finally, there exist non-decreasing 
functions $P: \mathbb{R}_{+} \rightarrow \mathbb{R}_{+}, G: \mathbb{R}_{+} \rightarrow \mathbb{R}_{+}$such that for every $\eta_{0} \in L^{\infty}\left([-r, 0) ; \mathbb{R}^{n}\right), y_{0} \in L^{\infty}\left([-r, 0) ; \mathbb{R}^{n}\right)$, $w \in L_{\mathrm{loc}}^{\infty}([-r,+\infty) ; W)$ it holds that:

$$
\left\|\eta_{t}-y_{t}\right\| \leq G(s(t)) \exp (P(s(t)) t)\left\|\eta_{0}-y_{0}\right\|, \text { for all } t \in[0, \delta)
$$

where $\delta=\min \left(t_{\max }\left(\eta_{0}, w\right), t_{\max }\left(y_{0}, w\right)\right), s(t):=\max \left(\sup _{0 \leq \tau \leq t}\left\|\eta_{\tau}\right\|, \sup _{0 \leq \tau \leq t}\left\|y_{\tau}\right\|, \sup _{0 \leq \tau \leq t}\left\|w_{\tau}\right\|\right)$ and $\eta \in L_{\mathrm{loc}}^{\infty}\left(\left[-r, t_{\max }\left(\eta_{0}, w\right)\right) ; \mathbb{R}^{n}\right), y \in L_{\mathrm{loc}}^{\infty}\left(\left[-r, t_{\max }\left(y_{0}, w\right)\right) ; \mathbb{R}^{n}\right)$ are the unique mappings satisfying $\eta(t)=$ $f\left(\eta_{t}, w_{t}\right)$ for $t \in\left[0, t_{\max }\left(\eta_{0}, w\right)\right)$ a.e., $y(t)=f\left(y_{t}, w_{t}\right)$ for $t \in\left[0, t_{\max }\left(y_{0}, u\right)\right)$ a.e. and $\eta(t)=\eta_{0}(t), y(t)=y_{0}(t)$ for $t \in[-r, 0)$ a.e..

Proof. Let arbitrary $\eta_{0} \in L^{\infty}\left([-r, 0) ; \mathbb{R}^{n}\right), w \in L_{\mathrm{loc}}^{\infty}([-r,+\infty) ; W)$. Define $s:=\max \left(\left\|\eta_{0}\right\|, \sup _{-r \leq t \leq r}|u(t)|\right)$ and select arbitrary $p \in \mathbb{R}^{n}$ with $|p| \leq s$. Define $\eta^{1}(t)=\eta_{0}(t)$ for $t \in[-r, 0)$ a.e. and $\eta^{1}(t)=p$ for $t \in[0, r)$. Clearly, $\eta^{1} \in L^{\infty}\left([-r, r) ; \mathbb{R}^{n}\right)$ with $\sup _{0 \leq \tau<r}\left\|\eta_{\tau}^{1}\right\| \leq s$

Without loss of generality we may assume that the non-decreasing function $a: \mathbb{R}_{+} \rightarrow \mathbb{R}_{+}$involved in $(2.2)$ satisfies $a(t) \geq t$ for all $t \geq 0$. Let $\delta \in(0, r)$ be such that:

$$
2 N(R) \delta<1, \text { for } R=5 a(s) .
$$

Using induction and assumption (H2), we can guarantee that the sequence of functions $\eta^{(k)}:[-r, \delta) \rightarrow \mathbb{R}^{n}$ for $k \geq 1$ defined by: $\eta^{(k)}(t)=\eta_{0}(t)$ for $t \in[-r, 0)$ a.e. and $\eta^{(k)}(t)=f\left(\eta_{t}^{(k-1)}, w_{t}\right)$ for $t \in[0, \delta)$ a.e. for $k \geq 2$ satisfies $\eta^{(k)} \in L^{\infty}\left([-r, \delta) ; \mathbb{R}^{n}\right)$ for $k \geq 1$.

Notice that (2.2) in conjunction with the facts that $\sup _{0 \leq \tau<r}\left\|\eta_{\tau}^{1}\right\| \leq s$ and $\sup _{0 \leq \tau \leq r}\left\|u_{\tau}\right\| \leq s$ implies that

$$
\sup _{0 \leq \tau<\delta}\left\|\eta_{\tau}^{1}\right\| \leq a(s) \text { and } \sup _{0 \leq \tau<\delta}\left\|\eta_{\tau}^{2}\right\| \leq a(s)
$$

We next claim that

$$
\max _{l=1, \ldots, k}\left(\sup _{0 \leq \tau<\delta}\left\|\eta_{\tau}^{(l)}\right\|\right) \leq 5 a(s) \text { for all } k \geq 1 .
$$

The proof of the claim is made by induction. We first notice that by virtue of (2.5) the claim is true for $k=1$ and $k=2$. Next suppose that the claim is true for certain $k \geq 2$. Notice that, by virtue of $(2.1)$ and the fact that $R=5 a(s)$, the following inequality holds for all $l=2, \ldots, k$ and for almost all $t \in[0, \delta)$ :

$$
\begin{aligned}
& \left|\eta^{(l+1)}(t)-\eta^{(l)}(t)\right|=\left|f\left(\eta_{t}^{(l)}, w_{t}\right)-f\left(\eta_{t}^{(l-1)}, w_{t}\right)\right| \\
& \leq N(R) \delta \sup _{-\delta \leq s<0}\left|\left(\eta_{t}^{(l)}\right)(s)-\left(\eta_{t}^{(l-1)}\right)(s)\right|+M(R) \sup _{-r \leq s<-\delta}\left|\left(\eta_{t}^{(l)}\right)(s)-\left(\eta_{t}^{(l-1)}\right)(s)\right| \\
& =N(R) \delta \sup _{-\delta \leq s<0}\left|\eta^{(l)}(t+s)-\eta^{(l-1)}(t+s)\right|+M(R) \sup _{-r \leq s<-\delta}\left|\eta^{(l)}(t+s)-\eta^{(l-1)}(t+s)\right| \\
& =N(R) \delta\left\|\eta^{(l)}-\eta^{(l-1)}\right\|_{[-r, \delta)} .
\end{aligned}
$$

Since (2.7) holds for almost all $t \in[0, \delta)$ and since $\eta^{(l+1)}(t)=\eta^{(l)}(t)=\eta_{0}(t)$ for almost all $t \in[-r, 0)$ and for all $l \geq 1$, we get:

$$
\left\|\eta^{(l+1)}-\eta^{(l)}\right\|_{[-r, \delta)} \leq N(R) \delta\left\|\eta^{(l)}-\eta^{(l-1)}\right\|_{[-r, \delta)}, \text { for all } l=2, \ldots, k \text {. }
$$


Inequality (2.8) implies that:

$$
\left\|\eta^{(2+m)}-\eta^{(1+m)}\right\|_{[-r, \delta)} \leq(N(R) \delta)^{m}\left\|\eta^{2}-\eta^{1}\right\|_{[-r, \delta)}, \text { for all } m=0, \ldots, k-1 .
$$

Using the triangle inequality repeatedly and the fact that $N(R) \delta \leq 1 / 2$ (a consequence of (2.4)), we get for all $l=2, \ldots, k$ :

$$
\begin{aligned}
& \left\|\eta^{(l+1)}-\eta^{(1)}\right\|_{[-r, \delta)} \leq \sum_{m=0}^{l-1}\left\|\eta^{(m+2)}-\eta^{(m+1)}\right\|_{[-r, \delta)} \\
& \leq\left\|\eta^{(2)}-\eta^{(1)}\right\|_{[-r, \delta)} \sum_{m=0}^{l-1}(N(R) \delta)^{m}=\left\|\eta^{(2)}-\eta^{(1)}\right\|_{[-r, \delta)} \frac{1-(N(R) \delta)^{l}}{1-N(R) \delta} \\
& \leq 2\left\|\eta^{(2)}-\eta^{(1)}\right\|_{[-r, \delta)} .
\end{aligned}
$$

It follows from (2.5) and (2.10) that

$$
\sup _{0 \leq \tau<\delta}\left\|\eta_{\tau}^{(k+1)}\right\|=\left\|\eta^{(k+1)}\right\|_{[-r, \delta)} \leq 5 a(s)
$$

and therefore we have proved (2.6).

The existence of $\eta \in L^{\infty}\left([-r, \delta) ; \mathbb{R}^{n}\right)$ satisfying $\eta(t)=f\left(\eta_{t}, w_{t}\right)$ for $t \in[0, \delta)$ a.e. and $\eta(t)=\eta_{0}(t)$ for $t \in[-r, 0)$ a.e. follows closely the proof of Banach's fixed point theorem on the closed set $L^{\infty}([-r, \delta) ; B)$, where $B \subseteq \mathbb{R}^{n}$ denotes the closed ball of radius $5 a(s)$. Since $(2.9)$ holds for all $m \geq 0$, the sequence $\eta^{(k)} \in$ $L^{\infty}([-r, \delta) ; B)$ is a Cauchy sequence. Therefore, there exists a unique limit $\eta \in L^{\infty}([-r, \delta) ; B)$. Assumption (H1) (inequality (2.1)) implies that the right hand side of the inequality

$$
\left|\eta(t)-f\left(\eta_{t}, w_{t}\right)\right| \leq\left|\eta(t)-\eta^{(k)}(t)\right|+\left|f\left(\eta_{t}, w_{t}\right)-f\left(\eta_{t}^{(k-1)}, w_{t}\right)\right|
$$

can be made arbitrarily small for sufficiently large $k \geq 1$ and for almost all $t \in[0, \delta)$ and consequently, we obtain $\eta(t)=f\left(\eta_{t}, w_{t}\right)$ for $t \in[0, \delta)$ a.e.

In order to prove uniqueness, we use a contradiction argument. Suppose that there exists $y \in L^{\infty}\left([-r, \delta) ; \mathbb{R}^{n}\right)$ satisfying $y(t)=f\left(y_{t}, w_{t}\right)$ for $t \in[0, \delta)$ a.e. and $y(t)=\eta_{0}(t)$ for $t \in[-r, 0)$ a.e. with $\sup _{-r \leq s<\delta}|y(s)-\eta(s)|>0$. Let $p \geq 0$ be the least upper bound of all $t \in[0, \delta)$ with $\sup _{-r \leq s<t}|y(s)-\eta(s)|=0$. A contradiction argument shows that $p<\delta$. Since $p \in[0, \delta)$ is the least upper bound of all $t \in[0, \delta)$ with $\sup _{-r \leq s<t}|y(s)-\eta(s)|=0$, it follows that $\sup _{-r \leq s<t}|y(s)-\eta(s)|>0$ for all $t \in(p, \delta)$. A contradiction argument shows that $\sup _{-r \leq s<p}|y(s)-\eta(s)|=0$. Define $R=\max \left(\sup _{-r \leq s<\delta}|y(s)|, \sup _{-r \leq s<\delta}|\eta(s)|, \sup _{-r \leq s \leq \delta}|w(s)|\right)$. It follows from (2.1) for almost all $t \in(p, \delta)$ and $h=t-p$ :

$$
|\eta(t)-y(t)|=\left|f\left(\eta_{t}, w_{t}\right)-f\left(y_{t}, w_{t}\right)\right| \leq N(R)(t-p) \sup _{p \leq s<t}|\eta(s)-y(s)| .
$$

Inequality (2.12) implies $\sup _{p<s<t}|\eta(s)-y(s)| \leq N(R)(t-p) \sup _{p \leq s<t}|\eta(s)-y(s)|$ for all $t \in(p, \delta)$ and since $\sup _{p<s<t}|\eta(s)-y(s)|=\sup _{p \leq s<t}|\eta(s)-y(s)|$, we obtain $\sup _{p \leq s<t}|\eta(s)-y(s)|=0$ for all $t<\min \left(\delta, p+\frac{1}{N(R)+1}\right)$, i.e., $\sup _{-r \leq s<t}|y(s)-\eta(s)|=0$ for all $t<\min \left(\delta, p+\frac{1}{N(R)+1}\right)$, a contradiction.

Since the unique function $\eta \in L^{\infty}\left([-r, \delta) ; \mathbb{R}^{n}\right)$ satisfying $\eta(t)=f\left(\eta_{t}, w_{t}\right)$ for $t \in[0, \delta)$ a.e. and $\eta(t)=\eta_{0}(t)$ for $t \in[-r, 0)$ a.e. is bounded, it follows (by repeating the arguments above) that there exists $\delta^{\prime}>\delta$ and a unique function $\eta \in L^{\infty}\left(\left[-r, \delta^{\prime}\right) ; \mathbb{R}^{n}\right)$ satisfying $\eta(t)=f\left(\eta_{t}, w_{t}\right)$ for $t \in\left[0, \delta^{\prime}\right)$ a.e. and $\eta(t)=\eta_{0}(t)$ for $t \in[-r, 0)$ a.e.. 
More specifically, by virtue of $(2.4), \delta^{\prime}>\delta$ satisfies

$$
\delta+r \geq \delta^{\prime} \geq \delta+\frac{r}{1+2 r N\left(5 a\left(\max \left(\sup _{-r \leq s<\delta}|\eta(s)|, \sup _{-r \leq s \leq \delta+r}|w(s)|\right)\right)\right) .}
$$

Let $t_{\max }=t_{\max }\left(\eta_{0}, w\right) \in(0,+\infty]$ be the least upper bound of all $\delta>0$ for which there exists function $\eta \in L^{\infty}\left([-r, \delta) ; \mathbb{R}^{n}\right)$ satisfying $\eta(t)=f\left(\eta_{t}, w_{t}\right)$ for $t \in[0, \delta)$ a.e. and $\eta(t)=\eta_{0}(t)$ for $t \in[-r, 0)$ a.e.. If $t_{\max }<+\infty$ (i.e., is finite) then for every $\varepsilon>0$ there exists $\delta>t_{\max }-\varepsilon$ and a function $\eta \in L^{\infty}\left([-r, \delta) ; \mathbb{R}^{n}\right)$ satisfying $\eta(t)=f\left(\eta_{t}, w_{t}\right)$ for $t \in[0, \delta)$ a.e. and $\eta(t)=\eta_{0}(t)$ for $t \in[-r, 0)$ a.e.. It follows in any case $\left(t_{\max }<+\infty\right.$ or $\left.t_{\max }=+\infty\right)$ that there exists a function $\eta \in L_{\mathrm{loc}}^{\infty}\left(\left[-r, t_{\max }\right) ; \mathbb{R}^{n}\right)$ satisfying $\eta(t)=f\left(\eta_{t}, w_{t}\right)$ for $t \in\left[0, t_{\max }\right)$ a.e. and $\eta(t)=\eta_{0}(t)$ for $t \in[-r, 0)$ a.e..

For the case $t_{\max }<+\infty$ we notice that (2.13) implies the inequality

$$
t_{\max } \geq \delta+\frac{r}{1+2 r N\left(5 a\left(\max \left(\sup _{-r \leq s<\delta}|\eta(s)|, \sup _{-r \leq s \leq \delta+r}|w(s)|\right)\right)\right)}
$$

or

$$
N\left(5 a\left(\max \left(\sup _{-r \leq s<\delta}|\eta(s)|, \sup _{-r \leq s \leq \delta+r}|w(s)|\right)\right)\right) \geq \frac{r-\varepsilon}{2 r \varepsilon}
$$

for all $\varepsilon>0$ and $\delta>t_{\max }-\varepsilon$. The above inequality cannot hold for all $\varepsilon>0$ if $\sup _{-r \leq s<t_{\max }}|\eta(s)|<+\infty$. Therefore, if $t_{\max }<+\infty$ then limsup $\left\|\eta_{t}\right\|=+\infty$.

$$
t \rightarrow t_{\max }^{-}
$$

Finally, we proceed to the constructive proof of estimate $(2.3)$. Let $\eta_{0} \in L^{\infty}\left([-r, 0) ; \mathbb{R}^{n}\right), y_{0} \in$ $L^{\infty}\left([-r, 0) ; \mathbb{R}^{n}\right), w \in L_{\mathrm{loc}}^{\infty}([-r,+\infty) ; W)$ and let $\eta \in L_{\mathrm{loc}}^{\infty}\left(\left[-r, t_{\max }\left(\eta_{0}, w\right)\right) ; \mathbb{R}^{n}\right), y \in L_{\mathrm{loc}}^{\infty}\left(\left[-r, t_{\max }\left(y_{0}, w\right)\right) ; \mathbb{R}^{n}\right)$ be the unique mappings satisfying $\eta(t)=f\left(\eta_{t}, w_{t}\right)$ for $t \in\left[0, t_{\max }\left(\eta_{0}, w\right)\right)$ a.e., $y(t)=f\left(y_{t}, w_{t}\right)$ for $t \in$ $\left[0, t_{\max }\left(y_{0}, w\right)\right)$ a.e. and $\eta(t)=\eta_{0}(t), y(t)=y_{0}(t)$ for $t \in[-r, 0)$ a.e.. Define $\delta=\min \left(t_{\max }\left(\eta_{0}, w\right), t_{\max }\left(y_{0}, w\right)\right)$. For all $t \in(0, \delta)$ we define $s(t):=\max \left(\sup _{0 \leq \tau \leq t}\left\|\eta_{\tau}\right\|, \sup _{0 \leq \tau \leq t}\left\|y_{\tau}\right\|, \sup _{0 \leq \tau \leq t}\left\|w_{\tau}\right\|\right)$ and $h=\frac{t}{N}$, where $N=$ $1+\left[\frac{t(1+2 r N(s(t)))}{\min (r, t)}\right]$ and $\left[\frac{t(1+2 r N(s(t)))}{\min (r, t)}\right]$ denotes the integer part of the real $\frac{t(1+2 r N(s(t)))}{\min (r, t)}$. Inequality (2.1) implies for almost all $v \in[0, h)$ :

$$
\begin{aligned}
& |\eta(v)-y(v)|=\left|f\left(\eta_{v}, w_{v}\right)-f\left(y_{v}, w_{v}\right)\right| \leq \\
& N(s(t)) h \sup _{-h \leq q<0}|\eta(v+q)-y(v+q)|+M(s(t))\left\|\eta_{0}-y_{0}\right\|
\end{aligned}
$$

Notice that since $N(s(t)) h \leq 1 / 2$ we get from $(2.14)$ :

$$
\sup _{-r \leq q<h}|\eta(q)-y(q)| \leq(1+2 M(s(t)))\left\|\eta_{0}-y_{0}\right\| .
$$

Using induction and similar arguments we can prove that the following inequality holds for $i=1, \ldots, N$

$$
\sup _{-r \leq q<i h}|\eta(q)-y(q)| \leq(1+2 M(s(t)))^{i}\left\|\eta_{0}-y_{0}\right\| .
$$

Since $N=1+\left[\frac{t(1+2 r N(s(t)))}{\min (r, t)}\right] \leq 1+\frac{t(1+2 r N(s(t)))}{\min (r, t)} \leq 2+2 r N(s(t))+\frac{t(1+2 r N(s(t)))}{r}$, we obtain from (2.16) for $i=N$ :

$$
\sup _{-r \leq q<t}|\eta(q)-y(q)| \leq(1+2 M(s(t)))^{2+2 r N(s(t))} \exp \left(\left(2 N(s(t))+r^{-1}\right) \ln (1+2 M(s(t))) t\right)\left\|\eta_{0}-y_{0}\right\| .
$$


Inequality (2.17) implies (2.3) with $G(s):=(1+2 M(s))^{2+2 r N(s)}$ and $P(s):=\left(2 N(s)+r^{-1}\right) \ln (1+2 M(s))$.

The proof is complete.

Having proved Theorem 2.1, we are justified for arbitrary $\eta_{0} \in L^{\infty}\left([-r, 0) ; \mathbb{R}^{n}\right), w \in L_{\text {loc }}^{\infty}([-r,+\infty) ; W)$ to name the unique mapping $\eta \in L_{\mathrm{loc}}^{\infty}\left(\left[-r, t_{\mathrm{max}}\right) ; \mathbb{R}^{n}\right)$ satisfying $\eta(t)=f\left(\eta_{t}, w_{t}\right)$ for $t \in\left[0, t_{\mathrm{max}}\right)$ a.e., $\eta(t)=\eta_{0}(t)$ for $t \in[-r, 0)$ a.e. for certain $t_{\max }=t_{\max }\left(\eta_{0}, w\right) \in(0,+\infty]$ as "the solution $\eta(t)$ of (1.1) with initial condition $\eta(t)=\eta_{0}(t)$ for $t \in[-r, 0)$ a.e., corresponding to input $w \in L_{\text {loc }}^{\infty}([-r,+\infty) ; W)$ ", without explicit reference to the maximal existence time of the solution $t_{\max }=t_{\max }\left(\eta_{0}, w\right) \in(0,+\infty]$.

A number of system-theoretic properties can be proved by using the results of Theorem 2.1. More specifically, we study system (1.1) under assumptions (H1), (H2) and the following assumption:

(H3) $w=(d, u)$, where $d \in D \subseteq \mathbb{R}^{m_{1}}$ is a compact set, $u \in U \subseteq \mathbb{R}^{m_{2}}$ is a closed set with $0 \in U$ and $m=m_{1}+m_{2}$. Moreover, there exists $b \in K_{\infty}$ such that for every $\eta \in L^{\infty}\left([-r, 0) ; \mathbb{R}^{n}\right), d \in L^{\infty}([-r, 0] ; D), u \in L^{\infty}([-r, 0] ; U)$, the following inequality holds:

$$
|f(\eta, d, u)| \leq b\left(\max \left(\|\eta\|, \sup _{-r \leq s \leq 0}|u(s)|\right)\right) .
$$

Assumption (H3) means that the system's right-hand side is uniformly bounded with respect to the disturbance $d$ and the right-hand side is zero when the state $\eta$ and input $u$ are zero, irrespective of the disturbance $d$. This means, in particular, that the origin is an equilibrium when the input $u$ is zero, irrespective of the value of the disturbance $d$, which excludes, for example, systems that have an additive disturbance in the model's right-hand side. In Example 2.7 we provide a simple system that captures some of the essence of assumption (H3) and the system's dependence on $d$.

We are ready to give a list of properties for system (1.1) that are derived from the results of Theorem 2.1.

Property 1: Robustness of the equilibrium point.

Assumption (H3) guarantees that $0 \in L^{\infty}\left([-r, 0) ; \mathbb{R}^{n}\right)$ is an equilibrium point for system (1.1), when $u$ is zero and for any $d$. However, in order to study the robust stability properties of the equilibrium point of (1.1) we need a stronger assertion, namely that $0 \in L^{\infty}\left([-r, 0) ; \mathbb{R}^{n}\right)$ is a robust equilibrium point for the system (1.1) with input $u \in U \subseteq \mathbb{R}^{m_{2}}$ (see [12]).

Theorem 2.2. Consider system (1.1) under assumptions (H1), (H2), (H3). The function $0 \in L^{\infty}\left([-r, 0) ; \mathbb{R}^{n}\right)$ is a robust equilibrium point for the system (1.1) with input $u \in U \subseteq \mathbb{R}^{m_{2}}$, i.e., for all $\varepsilon>0$ and $T \geq 0$ there exists $\delta:=\delta(\varepsilon, T)>0$ such that for every $\eta_{0} \in L^{\infty}\left([-r, 0) ; \mathbb{R}^{n}\right), d \in L^{\infty}([-r,+\infty) ; D), u \in L_{\mathrm{loc}}^{\infty}([-r,+\infty) ; U)$ with $\left\|\eta_{0}\right\|+\sup _{t \geq 0}\left\|u_{t}\right\|<\delta$ there exists $t_{\max }=t_{\max }\left(\eta_{0}, d, u\right) \in(T,+\infty]$ and a unique mapping $\eta \in L_{\mathrm{loc}}^{\infty}\left(\left[-r, t_{\max }\right) ; \mathbb{R}^{n}\right)$ satisfying $\eta(t)=f\left(\eta_{t}, d_{t}, u_{t}\right)$ for $t \in\left[0, t_{\max }\right)$ a.e., $\eta(t)=\eta_{0}(t)$ for $t \in[-r, 0)$ a.e. and $\left\|\eta_{t}\right\|<\varepsilon$ for all $t \in[0, T]$.

Proof. Since $D \subseteq \mathbb{R}^{m_{1}}$ is compact, we are in the position to define $Q:=\max _{d \in D}|d|$. Without loss of generality, we may assume that the function $b \in K_{\infty}$ involved in (2.18) satisfies $b(s) \geq s$ for all $s \geq 0$. Let arbitrary $\varepsilon>0$, $T \geq 0$ be given and define:

$$
\delta(\varepsilon, T):=\kappa^{-1}(\varepsilon / 2), \kappa:=g^{(l)}=\underbrace{g \circ \ldots \circ g}_{l \text { times }}, l=\left[\frac{T}{\rho}\right]+1 \text { and } \rho=\frac{r}{1+2 r N(5 b(Q+\varepsilon))}
$$

where $g(s):=5 b(s)$ for all $s \geq 0,\left[\frac{T}{\rho}\right]$ is the integer part of $\frac{T}{\rho}$ and $N: \mathbb{R}_{+} \rightarrow \mathbb{R}_{+}$is the function involved in (2.1). Let arbitrary $\eta_{0} \in L^{\infty}\left([-r, 0) ; \mathbb{R}^{n}\right), d \in L^{\infty}([-r,+\infty) ; D), u \in L_{\text {loc }}^{\infty}([-r,+\infty) ; U)$ with $\left\|\eta_{0}\right\|+\sup _{t>0}\left\|u_{t}\right\|<\delta$ and consider the unique mapping $\eta \in L_{\mathrm{loc}}^{\infty}\left(\left[-r, t_{\max }\right) ; \mathbb{R}^{n}\right)$ satisfying $\eta(t)=f\left(\eta_{t}, d_{t}, u_{t}\right)$ for $t \in\left[0, t_{\max }\right)$ a.e., 
$\eta(t)=\eta_{0}(t)$ for $t \in[-r, 0)$ a.e. for certain $t_{\max }=t_{\max }\left(\eta_{0}, d, u\right) \in(0,+\infty]$. Using $(2.18)$, the fact that $\sup _{t \geq 0}\left\|u_{t}\right\|<$ $\delta$, the fact that $\delta \leq \varepsilon$ (a direct consequence of definition (2.19)), the fact that $Q:=\max _{d \in D}|d|$ and proceeding exactly as in the proof of Theorem 2.1, we can show that:

"For all $t_{0} \in\left[0, t_{\max }\right)$ with $\left\|\eta_{t_{0}}\right\| \leq \varepsilon$ it holds that $\left\|\eta_{t}\right\| \leq g\left(\max \left(\left\|\eta_{t_{0}}\right\|, \delta\right)\right)$, for all $t \in\left[t_{0}, t_{0}+\rho\right] "$

We next claim that $\left\|\eta_{i \rho}\right\| \leq g^{(i)}(\delta)$, for all $i=1, \ldots, l\left(\left\|\eta_{i \rho}\right\|\right.$ is the norm $\left\|\eta_{t}\right\|$ for $\left.t=i \rho\right)$. The claim is a direct consequence of property (2.20) and definition (2.19). Therefore, we get $\left\|\eta_{t}\right\| \leq \kappa(\delta)$ for all $t \in[0, l \rho]$. Since $l \rho \geq T$ (a direct consequence of the fact that $l=\left[\frac{T}{\rho}\right]+1$; recall $(2.19)$ ) and $\delta=\kappa^{-1}(\varepsilon / 2)$ (recall (2.19)), we obtain $\left\|\eta_{t}\right\|<\varepsilon$ for all $t \in[0, T]$. The proof is complete.

Property 2: Robust global asymptotic stability and input-to-state stability

Using the results contained in [12], we are in a position to define the notion of (Uniform) Robust Global Asymptotic Stability and Input-to-State Stability (ISS) for system (1.1) under assumptions (H1), (H2), (H3). The notion of ISS for systems described by IDEs is completely analogous to the corresponding notion introduced by E. D. Sontag for finite-dimensional systems in [29].

Definition 2.3. Consider system (1.1) under assumptions (H1), (H2), (H3) and assume that for every $\eta_{0} \in$ $L^{\infty}\left([-r, 0) ; \mathbb{R}^{n}\right), d \in L^{\infty}([-r,+\infty) ; D), u \in L_{\text {loc }}^{\infty}([-r,+\infty) ; U)$, there exists $\eta \in L_{\text {loc }}^{\infty}\left([-r,+\infty) ; \mathbb{R}^{n}\right)$ satisfying $\eta(t)=f\left(\eta_{t}, d_{t}, u_{t}\right)$ for $t \in[0,+\infty)$ a.e., $\eta(t)=\eta_{0}(t)$ for $t \in[-r, 0)$ a.e.. We say that system (1.1) is Inputto-State Stable (ISS) from the input $u \in U \subseteq \mathbb{R}^{m_{2}}$ uniformly in $d \in D \subseteq \mathbb{R}^{m_{1}}$, if there exists a continuous, non-decreasing function $\gamma: \mathbb{R}_{+} \rightarrow \mathbb{R}_{+}$(called the gain function of the input $u \in L_{\text {loc }}^{\infty}([-r,+\infty) ; U)$ ) such that the following properties hold:

Robust Lagrange Stability: For every $\varepsilon>0$ it holds that

$$
\sup \left\{\left\|\eta_{t}\right\|-\sup _{0 \leq s \leq t} \gamma\left(\left\|u_{s}\right\|\right): t \geq 0,\left\|\eta_{0}\right\| \leq \varepsilon, d \in L^{\infty}([-r,+\infty) ; D), u \in L_{\mathrm{loc}}^{\infty}([-r,+\infty) ; U)\right\}<+\infty .
$$

Robust Lyapunov Stability: For every $\varepsilon>0$ there exists $\delta:=\delta(\varepsilon)>0$ such that

$$
\sup \left\{\left\|\eta_{t}\right\|-\sup _{0 \leq s \leq t} \gamma\left(\left\|u_{s}\right\|\right): t \geq 0,\left\|\eta_{0}\right\| \leq \delta, d \in L^{\infty}([-r,+\infty) ; D), u \in L_{\mathrm{loc}}^{\infty}([-r,+\infty) ; U)\right\}<\varepsilon .
$$

Uniform Robust Attractivity: For every $\varepsilon>0$ and $R \geq 0$ there exists $\tau:=\tau(\varepsilon, R)>0$ such that

$$
\sup \left\{\left\|\eta_{t}\right\|-\sup _{0 \leq s \leq t} \gamma\left(\left\|u_{s}\right\|\right): t \geq \tau,\left\|\eta_{0}\right\| \leq R, d \in L^{\infty}([-r,+\infty) ; D), u \in L_{\mathrm{loc}}^{\infty}([-r,+\infty) ; U)\right\}<\varepsilon .
$$

If $U=\{0\}$ then we say that $0 \in L^{\infty}\left([-r, 0) ; \mathbb{R}^{n}\right)$ is (Uniformly) Robustly Globally Asymptotically Stable (RGAS) for (1.1).

Lemma 2.1, Lemma 4.2 in [12] and Theorem 2.2 in [12] give us the following result.

Theorem 2.4. Consider system (1.1) under assumptions (H1), (H2), (H3). Then the following statements are equivalent:

(a) System (1.1) is ISS from the input $u \in U \subseteq \mathbb{R}^{m_{2}}$ uniformly in $d \in D \subseteq \mathbb{R}^{m_{1}}$.

(b) There exists $\sigma \in K L$ and a continuous, non-decreasing function $\gamma: \mathbb{R}_{+} \rightarrow \mathbb{R}_{+}$such that for every $\eta_{0} \in L^{\infty}\left([-r, 0) ; \mathbb{R}^{n}\right), d \in L^{\infty}([-r,+\infty) ; D), u \in L_{\mathrm{loc}}^{\infty}([-r,+\infty) ; U)$, there exists $\eta \in L_{\mathrm{loc}}^{\infty}\left([-r,+\infty) ; \mathbb{R}^{n}\right)$ satisfying $\eta(t)=f\left(\eta_{t}, d_{t}, u_{t}\right)$ for $t \in[0,+\infty)$ a.e., $\eta(t)=\eta_{0}(t)$ for $t \in[-r, 0)$ a.e. and $\left\|\eta_{t}\right\| \leq$ $\sigma\left(\left\|\eta_{0}\right\|, t\right)+\sup _{0 \leq s \leq t} \gamma\left(\left\|u_{s}\right\|\right)$ for all $t \geq 0$. 
Furthermore, if $U=\{0\}$ then the following statement is equivalent to statements (a), (b):

(c) System (1.1) is Robustly Forward Complete, i.e., for every $T \geq 0$ and $R \geq 0$ it holds that

$$
\sup \left\{\left\|\eta_{t}\right\|: t \in[0, T],\left\|\eta_{0}\right\| \leq R, d \in L^{\infty}([-r,+\infty) ; D)\right\}<+\infty
$$

where $\eta(t)$ denotes the solution of $(1.1)$ with $\eta(t)=\eta_{0}(t)$ for $t \in[-r, 0)$ a.e., corresponding to input $d \in$ $L^{\infty}([-r,+\infty) ; D)$, and the property of Uniform Robust Attractivity of Definition 2.3 holds.

Property 3: Lyapunov characterization of RGAS.

Theorem 3.4 in [12] and the results of Theorems 2.1, 2.2 allow us to obtain a complete Lyapunov characterization for the RGAS property for system (1.1).

Theorem 2.5. Consider system (1.1) under assumptions (H1), (H2), (H3) and assume that $U=\{0\}$. The equilibrium point $0 \in L^{\infty}\left([-r, 0) ; \mathbb{R}^{n}\right)$ is $R G A S$ for $(1.1)$ if and only if there exists a functional $V: L^{\infty}\left([-r, 0) ; \mathbb{R}^{n}\right) \rightarrow \mathbb{R}_{+}$, a non-decreasing function $Q: \mathbb{R}_{+} \rightarrow \mathbb{R}_{+}$and functions $a_{1}, a_{2} \in K_{\infty}$ such that the following inequalities hold:

$$
\begin{gathered}
a_{1}(\|\eta\|) \leq V(\eta) \leq a_{2}(\|\eta\|), \text { for all } \eta \in L^{\infty}\left([-r, 0) ; \mathbb{R}^{n}\right) \\
|V(\eta)-V(y)| \leq Q(\max (\|\eta\|,\|y\|))\|\eta-y\|, \text { for all } \eta, y \in L^{\infty}\left([-r, 0) ; \mathbb{R}^{n}\right) . \\
V\left(\eta_{t}\right) \leq \exp (-t) V\left(\eta_{0}\right), \text { for all } t \geq 0, \eta_{0} \in L^{\infty}\left([-r, 0) ; \mathbb{R}^{n}\right), d \in L^{\infty}([-r,+\infty) ; D)
\end{gathered}
$$

where $\eta_{t}$ denotes of (1.1) with initial condition $\eta(t)=\eta_{0}(t)$ for $t \in[-r, 0)$ a.e., corresponding to input $d \in$ $L^{\infty}([-r,+\infty) ; D)$.

Inequality (2.22) guarantees that the functional $V: L^{\infty}\left([-r, 0) ; \mathbb{R}^{n}\right) \rightarrow \mathbb{R}_{+}$is Lipschitz on bounded sets of the state space $L^{\infty}\left([-r, 0) ; \mathbb{R}^{n}\right)$. However, inequality $(2.22)$ does not guarantee Frechet differentiability of the functional $V: L^{\infty}\left([-r, 0) ; \mathbb{R}^{n}\right) \rightarrow \mathbb{R}_{+}$nor that the limit $\lim _{t \rightarrow 0^{+}} t^{-1}\left(V\left(\eta_{t}\right)-V\left(\eta_{0}\right)\right)$ exists for the solution $\eta(t)$ of (1.1) with initial condition $\eta(t)=\eta_{0}(t)$ for $t \in[-r, 0)$ a.e., corresponding to input $d \in L^{\infty}([-r,+\infty) ; D)$. Notice that inequality (2.23) guarantees that for every $\eta_{0} \in L^{\infty}\left([-r, 0) ; \mathbb{R}^{n}\right), d \in L^{\infty}([-r,+\infty) ; D)$, the solution $\eta(t)$ of (1.1) with initial condition $\eta(t)=\eta_{0}(t)$ for $t \in[-r, 0)$ a.e., corresponding to input $d \in L^{\infty}([-r,+\infty) ; D)$ satisfies $\limsup _{t \rightarrow 0^{+}} t^{-1}\left(V\left(\eta_{t}\right)-V\left(\eta_{0}\right)\right) \leq-V\left(\eta_{0}\right)$.

Property 4: Sufficient conditions for stability properties.

Theorem 2.5 is not the most convenient way of proving RGAS or ISS for (1.1). For practical purposes we can use the following result, which is an extension of the classical Razumikhin theorem for time delay systems $($ see $[10,12])$.

Theorem 2.6. Consider system (1.1) under assumptions (H1), (H2), (H3). Assume that there exists a continuous, positive definite and radially unbounded function $W: \mathbb{R}^{n} \rightarrow \mathbb{R}_{+}$, a continuous, non-decreasing function $\tilde{\gamma}: \mathbb{R}_{+} \rightarrow \mathbb{R}_{+}$and a constant $\lambda \in(0,1)$ such that the following inequality holds for all $\eta \in L^{\infty}\left([-r, 0) ; \mathbb{R}^{n}\right)$, $d \in L^{\infty}([-r, 0] ; D), u \in L^{\infty}([-r, 0] ; U):$

$$
W(f(\eta, d, u)) \leq \lambda \sup _{-r \leq s<0} W(\eta(s))+\tilde{\gamma}(\|u\|) .
$$

Then system (1.1) is ISS from the input $u \in U \subseteq \mathbb{R}^{m_{2}}$ uniformly in $d \in D \subseteq \mathbb{R}^{m_{1}}$ with gain function $\gamma(s):=a_{1}^{-1}\left(\frac{8}{(1-\lambda)^{2}(1+\lambda)} \tilde{\gamma}(s)\right)$ for all $s \geq 0$, where $a_{1} \in K_{\infty}$ satisfies $a_{1}(|\eta|) \leq W(\eta)$, for all $\eta \in \mathbb{R}^{n}$. 
Proof. Define the functional:

$$
V(\eta):=\sup _{-r \leq s<0} \exp (\sigma s) W(\eta(s)), \text { for all } \eta \in L^{\infty}\left([-r, 0) ; \mathbb{R}^{n}\right)
$$

where $\sigma \in\left(0, \frac{1}{r} \ln \left(\frac{1}{\lambda}\right)\right)$ is a constant. Since $W: \mathbb{R}^{n} \rightarrow \mathbb{R}_{+}$is a continuous, positive definite and radially unbounded function, there exist functions $a_{1}, a_{2} \in K_{\infty}$ such that:

$$
a_{1}(|\eta|) \leq W(\eta) \leq a_{2}(|\eta|), \text { for all } \eta \in \mathbb{R}^{n}
$$

Using (2.25) and (2.26) we conclude that the following inequality holds:

$$
\tilde{a}_{1}(\|\eta\|) \leq V(\eta) \leq a_{2}(\|\eta\|), \text { for all } \eta \in L^{\infty}\left([-r, 0) ; \mathbb{R}^{n}\right)
$$

where $\tilde{a}_{1}(s):=\exp (-\sigma r) a_{1}(s)$, for all $s \geq 0$.

Let arbitrary $\eta_{0} \in L^{\infty}\left([-r, 0) ; \mathbb{R}^{n}\right), d \in L^{\infty}([-r,+\infty) ; D), u \in L^{\infty}([-r,+\infty) ; U)$ and consider the unique solution $\eta(t)$ of $(1.1)$ with initial condition $\eta(t)=\eta_{0}(t)$ for $t \in[-r, 0)$ a.e., corresponding to inputs $d \in$ $L^{\infty}([-r,+\infty) ; D), u \in L_{\text {loc }}^{\infty}([-r,+\infty) ; U)$. Let arbitrary $t \in\left(0, t_{\max }\left(\eta_{0}, d, u\right)\right)$, where $t_{\max }\left(\eta_{0}, d, u\right)>0$ is the maximal existence time of the solution and let $h \in\left(0, t_{\max }\left(\eta_{0}, d, u\right)-t\right)$. Using definition $(2.25)$ we get:

$$
\begin{aligned}
& V\left(\eta_{t+h}\right):=\sup _{-r \leq s<0} \exp (\sigma s) W(\eta(t+h+s)) \\
& =\max \left(\sup _{-r \leq s<-h} \exp (\sigma s) W(\eta(t+h+s)), \sup _{-h \leq s<0} \exp (\sigma s) W(\eta(t+h+s))\right) \\
& =\max \left(\exp (-\sigma h) \sup _{-r+h \leq s<0} \exp (\sigma s) W(\eta(t+s)), \sup _{-h \leq s<0} \exp (\sigma s) W(\eta(t+h+s))\right) \\
& \leq \max \left(\exp (-\sigma h) V\left(\eta_{t}\right), \sup _{0 \leq s<h} W(\eta(t+s))\right) .
\end{aligned}
$$

Using (2.24) we get for almost all $q \in[0, h)$ :

$$
W(\eta(t+q))=W\left(f\left(\eta_{t+q}, d_{t+q}, u_{t+q}\right)\right) \leq \lambda \max \left(\sup _{-r+q \leq s<0} W(\eta(t+s)), \sup _{0 \leq s<q} W(\eta(t+s))\right)+\tilde{\gamma}(\|u\|)
$$

where $\|u\|=\sup _{t \geq 0}\left\|u_{t}\right\|$. Definition (2.25) and inequality (2.29) gives:

$$
\sup _{0 \leq q<h} W(\eta(t+q)) \leq \lambda \max \left(\exp (\sigma r) V\left(\eta_{t}\right), \sup _{0 \leq s<h} W(\eta(t+s))\right)+\tilde{\gamma}(\|u\|)
$$

which directly implies:

$$
\sup _{0 \leq q<h} W(\eta(t+q)) \leq \lambda \exp (\sigma r) V\left(\eta_{t}\right)+\frac{1}{1-\lambda} \tilde{\gamma}(\|u\|) .
$$

Since $\lambda \exp (\sigma r) \leq \exp (-\sigma h)$ for all $h \leq \frac{1}{\sigma} \ln \left(\frac{1}{\lambda}\right)-r$ (notice that $\sigma \in\left(0, \frac{1}{r} \ln \left(\frac{1}{\lambda}\right)\right)$ ), we get from (2.28), (2.30) for all $h \in\left(0, t_{\max }\left(\eta_{0}, d, u\right)-t\right)$ with $h \leq \frac{1}{\sigma} \ln \left(\frac{1}{\lambda}\right)-r$ :

$$
V\left(\eta_{t+h}\right) \leq \exp (-\sigma h) V\left(\eta_{t}\right)+\frac{1}{1-\lambda} \tilde{\gamma}(\|u\|) .
$$

Using the Boundedness-Implies-Continuation property, inequalities (2.27), (2.31) and a standard contradiction argument, we conclude that $t_{\max }\left(\eta_{0}, d, u\right)=+\infty$. Therefore, we conclude that (2.31) holds for all $t \geq 0$ and $h \geq 0$ with $h \leq \frac{1}{\sigma} \ln \left(\frac{1}{\lambda}\right)-r$. 
Using induction and (2.31) we show that $V\left(\eta_{i h}\right) \leq \exp (-i \sigma h) V\left(\eta_{0}\right)+\frac{1}{1-\lambda} \tilde{\gamma}(\|u\|) \sum_{j=0}^{i-1} \exp (-j \sigma h)$, for all integers $i \geq 1$ and $h=\frac{1}{\sigma} \ln \left(\frac{1}{\lambda}\right)-r$. Consequently, the previous inequality in conjunction with (2.31) shows that $V\left(\eta_{i h+q}\right) \leq \exp (-\sigma(q+i h)) V\left(\eta_{0}\right)+\frac{1}{1-\lambda} \tilde{\gamma}(\|u\|) \frac{2-\exp (-\sigma h)}{1-\exp (-\sigma h)}$, for all integers $i \geq 0, h=\frac{1}{\sigma} \ln \left(\frac{1}{\lambda}\right)-r$ and for all $q \in\left[0, \frac{1}{\sigma} \ln \left(\frac{1}{\lambda}\right)-r\right)$. Since, for every $t \geq 0$ there exists integer $i \geq 0$ and $q \in\left[0, \frac{1}{\sigma} \ln \left(\frac{1}{\lambda}\right)-r\right)$ with $t=i h+q$, we obtain:

$$
V\left(\eta_{t}\right) \leq \exp (-\sigma t) V\left(\eta_{0}\right)+\frac{1}{1-\lambda} \frac{2-\lambda \exp (\sigma r)}{1-\lambda \exp (\sigma r)} \tilde{\gamma}(\|u\|), \text { for all } t \geq 0 .
$$

Inequality (2.32) in conjunction with (2.27) gives for all $\varepsilon>0$ :

$$
\tilde{a}_{1}\left(\left\|\eta_{t}\right\|\right) \leq \max \left((1+\varepsilon) \exp (-\sigma t) a_{2}\left(\left\|\eta_{0}\right\|\right), \frac{1+\varepsilon^{-1}}{1-\lambda} \frac{2-\lambda \exp (\sigma r)}{1-\lambda \exp (\sigma r)} \tilde{\gamma}(\|u\|)\right), \text { for all } t \geq 0 .
$$

Inequality (2.33) with $\varepsilon=1$ and $\sigma=\frac{1}{r} \ln \left(\frac{2}{1+\lambda}\right)$ shows that there exists $\sigma \in K L$ such that for every $\eta_{0} \in L^{\infty}\left([-r, 0) ; \mathbb{R}^{n}\right), d \in L^{\infty}([-r,+\infty) ; D), u \in L^{\infty}([-r,+\infty) ; U)$, there exists $\eta \in L_{\text {loc }}^{\infty}\left([-r,+\infty) ; \mathbb{R}^{n}\right)$ satisfying $\eta(t)=f\left(\eta_{t}, d_{t}, u_{t}\right)$ for $t \in[0,+\infty)$ a.e., $\eta(t)=\eta_{0}(t)$ for $t \in[-r, 0)$ a.e. and $\left\|\eta_{t}\right\| \leq \sigma\left(\left\|\eta_{0}\right\|, t\right)+\gamma(\|u\|)$ for all $t \geq 0$ with $\gamma(s):=a_{1}^{-1}\left(\frac{8}{(1-\lambda)^{2}(1+\lambda)} \tilde{\gamma}(s)\right)$ for all $s \geq 0$. A standard causality argument shows that the estimate $\left\|\eta_{t}\right\| \leq \sigma\left(\left\|\eta_{0}\right\|, t\right)+\sup _{0 \leq s \leq t} \gamma\left(\left\|u_{s}\right\|\right)$ holds for all $t \geq 0$ and $u \in L_{\text {loc }}^{\infty}([-r,+\infty) ; U)$. Therefore, Theorem 2.4 implies that system (1.1) is ISS from the input $u \in U \subseteq \mathbb{R}^{m_{2}}$ uniformly in $d \in D \subseteq \mathbb{R}^{m_{1}}$ with gain function $\gamma(s):=a_{1}^{-1}\left(\frac{8}{(1-\lambda)^{2}(1+\lambda)} \tilde{\gamma}(s)\right)$ for all $s \geq 0$. The proof is complete.

Example 2.7. Consider the linear system described by the single IDE:

$$
\begin{aligned}
& \eta(t)=d(t) \int_{-1}^{0} q(s) \eta(t+s) \mathrm{d} s+u(t) \\
& \eta(t) \in \mathbb{R}, d(t) \in[-1,1], u(t) \in \mathbb{R}
\end{aligned}
$$

where $q:[-1,0] \rightarrow \mathbb{R}$ is a continuous function. Using Theorem 2.6 with $W(\eta)=|\eta|$, we can conclude that system (2.34) is ISS from the input $u \in \mathbb{R}$ under the assumption that there exists $\lambda \in(0,1)$ such that $\int_{-1}^{0}|q(s)| \mathrm{d} s \leq \lambda$. The gain function of the input $u \in \mathbb{R}$ is estimated by Theorem 2.6 to be the linear function $\gamma(s):=\frac{8}{(1-\lambda)^{2}(1+\lambda)} s$ for all $s \geq 0$.

\section{SySTEMS DESCRIBED BY A SINGLE FIRST-ORDER HYPERBOLIC PARTIAL DIFFERENTIAL EQUATION}

Consider the system described by a single FOH-PDE of the form (1.2), (1.3), (1.4) under the following assumption:

(A1) There exist non-decreasing functions $L: \mathbb{R}_{+} \rightarrow \mathbb{R}_{+}, \sigma: \mathbb{R}_{+} \rightarrow \mathbb{R}_{+}$such that the functionals $G: \mathbb{R}^{m} \times$ $L^{\infty}((0,1] ; \mathbb{R}) \rightarrow \mathbb{R}, K_{i}: \mathbb{R}^{m} \times L^{\infty}((0,1] ; \mathbb{R}) \rightarrow \mathbb{R}(i=1, \ldots, N)$ satisfy the following inequalities for all $R \geq 0:$

$$
\begin{aligned}
& |G(w, x)-G(w, y)|+\sum_{i=1}^{N}\left|K_{i}(w, x)-K_{i}(w, y)\right| \leq L(R) h \sup _{0<z \leq h}(|x(z)-y(z)|)+L(R) \sup _{h<z \leq 1}(|x(z)-y(z)|), \\
& \quad \text { for all } h \in(0,1), w \in W \text { with }|w| \leq R \text { and } x, y \in L^{\infty}((0,1] ;[-R, R]) \\
& |G(w, x)|+\sum_{i=1}^{N}\left|K_{i}(w, x)\right| \leq \sigma(R), \text { for all } w \in W \text { with }|w| \leq R \text { and } x \in L^{\infty}((0,1] ;[-R, R]) .
\end{aligned}
$$

We show next how to transform system (1.2), (1.3), (1.4) under assumption (A1) to an equivalent system described by IDEs. 
Consider a classical solution of (1.2), (1.3), (1.4) with sufficiently regular initial condition $x(0, z)=x_{0}(z)$ for $z \in(0,1]$ corresponding to a sufficiently regular input $w: \mathbb{R}_{+} \rightarrow W$. We assume that the solution exists for all $t \in J$, where $J \subseteq \mathbb{R}_{+}$is an interval with non-empty interior and $0 \in J$. First, we define for $t \in J$ :

$$
v(t)=G\left(w(t), x_{t}\right) .
$$

If we define for almost all $q \in\left(0, c^{-1}\right]$ :

$$
v(-q)=\exp \left(-\int_{-q}^{0} a(p(s), c(q+s)) \mathrm{d} s\right) x_{0}(c q)-\int_{-q}^{0} \exp \left(-\int_{-q}^{\tau} a(p(s), c(q+s)) \mathrm{d} s\right) g(c(q+\tau)) p(\tau) \mathrm{d} \tau
$$

then integration on the characteristic line allows us to conclude that the solution of (1.2), (1.3), (1.4) must satisfy the following equation for all $t \in J$ and $z \in[0,1]$ :

$$
\begin{aligned}
x(t, z)= & \exp \left(\int_{t-c^{-1} z}^{t} a(p(s), z+c(s-t)) \mathrm{d} s\right) v\left(t-c^{-1} z\right) \\
& +\int_{t-c^{-1} z}^{t} \exp \left(\int_{\tau}^{t} a(p(s), z+c(s-t)) \mathrm{d} s\right) g(z+c(\tau-t)) p(\tau) \mathrm{d} \tau .
\end{aligned}
$$

Let $p_{t} \in L^{\infty}\left(\left[-c^{-1}, 0\right) ; \mathbb{R}^{N}\right)$ be the mapping defined by $\left(p_{t}\right)(s)=p(t+s)$ for $t \in J, s \in\left[-c^{-1}, 0\right)$. Similarly, let $v_{t} \in L^{\infty}\left(\left[-c^{-1}, 0\right) ; \mathbb{R}\right)$ be the mapping defined by $\left(v_{t}\right)(s)=v(t+s)$ for $t \in J, s \in\left[-c^{-1}, 0\right)$. Clearly, equation (3.5) allows us to define for every $p \in L^{\infty}\left(\left[-c^{-1}, 0\right) ; \mathbb{R}^{N}\right)$ a bounded linear operator $A(p): L^{\infty}\left(\left[-c^{-1}, 0\right) ; \mathbb{R}\right) \rightarrow L^{\infty}((0,1] ; \mathbb{R})$ and a mapping $B: L^{\infty}\left(\left[-c^{-1}, 0\right) ; \mathbb{R}^{N}\right) \rightarrow L^{\infty}((0,1] ; \mathbb{R})$, so that the following equation holds for all $t \in J$ :

$$
\begin{gathered}
x_{t}=A\left(p_{t}\right) v_{t}+B\left(p_{t}\right) \\
(A(p) v)(z):=\exp \left(\int_{-c^{-1} z}^{0} a(p(t+q), z+c q) \mathrm{d} q\right) v\left(-c^{-1} z\right) \\
(B(p))(z):=\int_{-c^{-1} z}^{0} \exp \left(\int_{s}^{0} a(p(q), z+c q) \mathrm{d} q\right) g(z+c s) p(s) \mathrm{d} s, \text { for } z \in(0,1] .
\end{gathered}
$$

It follows from (3.3) and (3.6a) that the following equations hold for all $t \in J$ :

$$
p_{i}(t)=F_{i}\left(w(t), p_{t}, v_{t}\right), \text { for } i=1, \ldots, N \text { and } v(t)=F_{N+1}\left(w(t), p_{t}, v_{t}\right)
$$

where the mappings $F_{i}: W \times L^{\infty}\left(\left[-c^{-1}, 0\right) ; \mathbb{R}^{N+1}\right) \rightarrow \mathbb{R}(i=1, \ldots, N+1)$ are defined for every $(w, p, v) \in$ $W \times L^{\infty}\left(\left[-c^{-1}, 0\right) ; \mathbb{R}^{N+1}\right)$ by the equations:

$$
F_{i}(w, p, v)=K_{i}(w, A(p) v+B(p)), \text { for } i=1, \ldots, N \text { and } F_{N+1}(w, p, v)=G(w, A(p) v+B(p)) .
$$

In other words, every classical solution $x_{t}$ of $(1.2),(1.3),(1.4)$ corresponds to a solution $\left(p_{t}, v_{t}\right) \in$ $L^{\infty}\left(\left[-c^{-1}, 0\right) ; \mathbb{R}^{N+1}\right)$ of the system described by the integral delay equations $(3.7),(3.8)$.

The procedure described above is rigorous and the system described by the IDEs (3.7), (3.8) is equivalent to the system described by the single FOH-PDE (1.2), (1.3), (1.4). This is shown by the following theorem. The key idea in Theorem 3.1 is to bypass the existence problem for the solutions of (1.2), (1.3), (1.4) by studying the system of IDEs (3.7). However, we first have to generalize the notion of the solution of the (1.2), (1.3), (1.4).

A solution of $(1.2),(1.3),(1.4)$ on the interval $[0, T)$, where $T>0$, can be any mapping $t \rightarrow x_{t} \in L^{\infty}([0,1] ; \mathbb{R})$ which satisfies $(1.3),(1.4),(3.3)$ for $t \in[0, T)$ a.e. with $(p, v) \in L_{\text {loc }}^{\infty}\left([0, T) ; \mathbb{R}^{N+1}\right)$, and

$$
\begin{aligned}
x(t, z)= & \int_{\max \left(0, t-c^{-1} z\right)}^{t} \exp \left(\int_{\tau}^{t} a(p(s), z+c(s-t)) \mathrm{d} s\right) g(z+c(\tau-t)) p(\tau) \mathrm{d} \tau \\
& +\exp \left(\int_{\max \left(0, t-c^{-1} z\right)}^{t} a(p(s), z+c(s-t)) \mathrm{d} s\right)\left\{\begin{array}{ccc}
v\left(t-c^{-1} z\right) & \text { for } \quad z \leq c t \\
x_{0}(z-c t) & \text { for } \quad z>c t
\end{array}\right.
\end{aligned}
$$


for all $t \in[0, T)$ and almost all $z \in[0,1]$. This is a notion of a solution which is close to the notion of the "mild" solution for first-order systems of hyperbolic PDEs (see [9] and references therein). A solution of system (3.7) gives us directly (by means of $(3.4)$ and $(3.5))$ a locally bounded mapping $\left[0, t_{\max }\right)$ э $t \rightarrow x_{t} \in L^{\infty}([0,1] ; \mathbb{R})$ satisfying $(1.3),(1.4),(3.3)$ for $t \in\left[0, t_{\max }\right)$ a.e. with $(p, v) \in L_{\mathrm{loc}}^{\infty}\left(\left[0, t_{\max }\right) ; \mathbb{R}^{N+1}\right)$, and (3.9) for all $t \in\left[0, t_{\max }\right)$ and almost all $z \in[0,1]$. This is the "mild" solution of the PDE (1.2), (1.3), (1.4). Uniqueness is established by exploiting the classical semigroup property of the solution mapping for (1.2), (1.3), (1.4).

Theorem 3.1. Assume that $a \in C^{0}\left(\mathbb{R}^{N} \times[0,1] ; \mathbb{R}\right)$ is locally Lipschitz and $g_{i} \in C^{0}([0,1] ; \mathbb{R})(i=1, \ldots, N)$. Moreover, assume that there exist non-decreasing functions $L: \mathbb{R}_{+} \rightarrow \mathbb{R}_{+}, \sigma: \mathbb{R}_{+} \rightarrow \mathbb{R}_{+}$such that the functionals $G: W \times L^{\infty}((0,1] ; \mathbb{R}) \rightarrow \mathbb{R}, K_{i}: W \times L^{\infty}((0,1] ; \mathbb{R}) \rightarrow \mathbb{R}(i=1, \ldots, N)$ satisfy inequalities (3.1), (3.2) for all $R \geq 0$. Finally, let the following assumption hold for the mapping $F: W \times L^{\infty}\left(\left[-c^{-1}, 0\right) ; \mathbb{R}^{N+1}\right) \rightarrow \mathbb{R}^{N+1}$, where $F(w, p, v):=\left(F_{1}(w, p, v), \ldots, F_{N+1}(w, p, v)\right)$ and $F_{i}: W \times L^{\infty}\left(\left[-c^{-1}, 0\right) ; \mathbb{R}^{N+1}\right) \rightarrow \mathbb{R}(i=1, \ldots, N+1)$ are defined by (3.8) with the aid of $(3.6 \mathrm{~b})$.

(A2) For every $\delta>0,(p, v) \in L^{\infty}\left(\left[-c^{-1}, \delta\right) ; \mathbb{R}^{N+1}\right), w \in L^{\infty}([0, \delta] ; W)$, every function $\xi:\left[-c^{-1}, \delta\right) \rightarrow \mathbb{R}^{N+1}$ defined by $\xi(t)=(p(t), v(t))$ for $t \in\left[-c^{-1}, 0\right)$ a.e. and $\xi(t)=F\left(w(t), p_{t}, v_{t}\right)$ for $t \in[0, \delta)$ a.e. satisfies $\xi \in$ $L^{\infty}\left(\left[-c^{-1}, \delta\right) ; \mathbb{R}^{N+1}\right)$.

Then for every $x_{0} \in L^{\infty}([0,1] ; \mathbb{R}), w \in L_{\mathrm{loc}}^{\infty}([0,+\infty) ; W)$ there exists $t_{\max }=t_{\max }\left(x_{0}, w\right) \in(0,+\infty]$ and a unique locally bounded mapping $\left[0, t_{\max }\right)$ э $t \rightarrow x_{t} \in L^{\infty}([0,1] ; \mathbb{R})$ satisfying $(1.3)$, (1.4), (3.3) for $t \in\left[0, t_{\max }\right)$ a.e. with $(p, v) \in L_{\mathrm{loc}}^{\infty}\left(\left[0, t_{\max }\right) ; \mathbb{R}^{N+1}\right)$, and (3.9) for all $t \in\left[0, t_{\max }\right)$ and almost all $z \in[0,1]$. The mapping $t \rightarrow x_{t} \in L^{\infty}([0,1] ; \mathbb{R})$ is obtained from (3.5) and the solution of the system (3.7) with arbitrary initial condition $(p, v) \in L^{\infty}\left(\left[-c^{-1}, 0\right) ; \mathbb{R}^{N+1}\right)$ that satisfies (3.4). Moreover, if $t_{\max }<+\infty$ then $\lim \sup \sup _{0 \leq z \leq 1}\left|\left(x_{t}\right)(z)\right|=+\infty$. $t \rightarrow t_{\max }^{-} 0 \leq z \leq 1$

The reader should notice that no regularity result is provided for the mapping $t \rightarrow x_{t} \in L^{\infty}([0,1] ; \mathbb{R})$. It is clear that the unique mapping $t \rightarrow x_{t} \in L^{\infty}([0,1] ; \mathbb{R})$ satisfying (1.3), (1.4), (3.3) for $t \in\left[0, t_{\max }\right)$ a.e. with $(p, v) \in L_{\text {loc }}^{\infty}\left(\left[0, t_{\max }\right) ; \mathbb{R}^{N+1}\right),(3.9)$ for all $t \in\left[0, t_{\max }\right)$ and almost all $z \in[0,1]$ is not a classical solution of the initial value problem (1.2), (1.3), (1.4) with initial condition $x(0, z)=x_{0}(z)$ for $z \in[0,1]$ a.e., where $x_{0} \in L^{\infty}([0,1] ; \mathbb{R})$.

The time $t_{\max }=t_{\max }\left(x_{0}, w\right) \in(0,+\infty]$ is the maximal existence time of the "mild" solution of the PDE (1.2), (1.3), (1.4). Indeed, Theorem 3.1 guarantees that a finite value for $t_{\max }$ implies "blow up" of the solution (a "finite escape time") exactly as in the case of systems described by ODEs (see [8]), systems described by Retarded Functional Differential Equations (see [10]) and systems described by IDEs (see Thm. 2.1 above). Therefore, the system (1.2), (1.3), (1.4) satisfies the Boundedness-Implies-Continuation property (see [12]).

The "mild" solution of the PDE (1.2), (1.3), (1.4) satisfies additional properties. It depends continuously on the initial conditions and is a continuous mapping when embedded in the spaces $L^{\mu}([0,1] ; \mathbb{R})$ for $\mu \in[1,+\infty)$. This is guaranteed by the following result.

Theorem 3.2. Consider system (1.3), (1.4), (3.3) under all assumptions made in Theorem 3.1. Then for every $x_{0} \in L^{\infty}([0,1] ; \mathbb{R}), w \in L_{\mathrm{loc}}^{\infty}([0,+\infty) ; W)$ the unique locally bounded mapping $\left[0, t_{\max }\right) \ni t \rightarrow x_{t} \in L^{\infty}([0,1] ; \mathbb{R})$ satisfying (1.3), (1.4), (3.3) for $t \in\left[0, t_{\max }\right)$ a.e. with $(p, v) \in L_{\mathrm{loc}}^{\infty}\left(\left[0, t_{\max }\right) ; \mathbb{R}^{N+1}\right)$, and (3.9) for all $t \in\left[0, t_{\max }\right)$ and almost all $z \in[0,1]$ is a continuous mapping $t \rightarrow x_{t} \in L^{\mu}([0,1] ; \mathbb{R})$ on $\left[0, t_{\max }\right)$ for every $\mu \in[1,+\infty)$. Finally, there exist non-decreasing functions $P: \mathbb{R}_{+} \rightarrow \mathbb{R}_{+}, Q: \mathbb{R}_{+} \rightarrow \mathbb{R}_{+}$such that for every $x_{0} \in L^{\infty}([0,1] ; \mathbb{R})$, $y_{0} \in L^{\infty}([0,1] ; \mathbb{R}), w \in L_{\mathrm{loc}}^{\infty}([0,+\infty) ; W)$ it holds that:

$$
\sup _{0 \leq z \leq 1}\left|\left(x_{t}\right)(z)-\left(y_{t}\right)(z)\right| \leq Q(s(t)) \exp (P(s(t)) t) \sup _{0 \leq z \leq 1}\left|\left(x_{0}\right)(z)-\left(y_{0}\right)(z)\right| \text {, for all } t \in[0, \delta)
$$

where $\delta=\min \left(t_{\max }\left(x_{0}, w\right), t_{\max }\left(y_{0}, w\right)\right), s(t):=\max \left(\sup _{0 \leq \tau<t} \sup _{0 \leq z \leq 1}\left|\left(x_{\tau}\right)(z)\right|, \sup _{0 \leq \tau<t} \sup _{0 \leq z \leq 1}\left|\left(y_{\tau}\right)(z)\right|, \sup _{0 \leq \tau \leq t}|w(t)|\right)$ and the mappings $t \rightarrow x_{t} \in L^{\infty}([0,1] ; \mathbb{R}), t \rightarrow y_{t} \in L^{\infty}([0,1] ; \mathbb{R})$ are the unique mappings satisfying (1.3), (1.4), (3.3) for $t \in\left[0, t_{\max }\right)$ a.e. with $(p, v) \in L_{\mathrm{loc}}^{\infty}\left(\left[0, t_{\max }\right) ; \mathbb{R}^{N+1}\right)$, (3.9) for all $t \in\left[0, t_{\max }\right)$ and almost all $z \in[0,1]$ as well as the analogous equations with $y$ replacing $x$. 
The proofs of Theorems 3.1 and 3.2 are provided at the Appendix.

Having stated Theorems 3.1 and 3.2 , we are justified for arbitrary $x_{0} \in L^{\infty}([0,1] ; \mathbb{R}), w \in L_{\mathrm{loc}}^{\infty}([0,+\infty) ; W)$ to name the unique mapping $\left[0, t_{\max }\right)$ э $t \rightarrow x_{t} \in L^{\infty}([0,1] ; \mathbb{R})$ satisfying $(1.3),(1.4),(3.3)$ for $t \in\left[0, t_{\max }\right)$ a.e. with $(p, v) \in L_{\text {loc }}^{\infty}\left(\left[0, t_{\max }\right) ; \mathbb{R}^{N+1}\right)$, and (3.9) for all $t \in\left[0, t_{\max }\right)$ and almost all $z \in[0,1]$ as "the solution $x_{t}$ of (1.2), (1.3), (1.4) with initial condition $x_{0} \in L^{\infty}([0,1] ; \mathbb{R})$, corresponding to input $w \in L_{\text {loc }}^{\infty}([0,+\infty) ; W)$ ", without explicit reference to the maximal existence time of the solution $t_{\max }=t_{\max }\left(x_{0}, w\right) \in(0,+\infty]$.

A number of system-theoretic properties can be proved by using the results of Theorems 3.1 and 3.2. More specifically, we study system (1.2), (1.3), (1.4) under assumptions (A1), (A2) and the following assumption:

(A3) $w=(d, u)$, where $d \in D \subseteq \mathbb{R}^{m_{1}}$ is a compact set, $u \in U \subseteq \mathbb{R}^{m_{2}}$ is a closed set with $0 \in U$ and $m=m_{1}+m_{2}$. Moreover, there exists $b \in K_{\infty}$ such that for every $x \in L^{\infty}([0,1] ; \mathbb{R}), d \in D, u \in U$, the following inequality holds:

$$
|G(d, u, x)|+\sum_{i=1}^{N}\left|K_{i}(d, u, x)\right| \leq b\left(\max \left(|u|, \sup _{0 \leq z \leq 1}|x(z)|\right)\right) .
$$

We are ready to give a list of properties for system (1.2), (1.3), (1.4) that are derived from the results of Theorems 3.1 and 3.2 .

Property 1: Robustness of the equilibrium point.

Assumption (A3) guarantees that $0 \in L^{\infty}([0,1] ; \mathbb{R})$ is an equilibrium point for system (1.2), (1.3), (1.4), when $u \in U \subseteq \mathbb{R}^{m_{2}}$ is zero for any $d \in D \subseteq \mathbb{R}^{m_{1}}$. However, in order to study the robust stability properties of the equilibrium point of $(1.2),(1.3),(1.4)$ we need a stronger assertion, namely that $0 \in L^{\infty}([0,1] ; \mathbb{R})$ is a robust equilibrium point for the system (1.2), (1.3), (1.4) with input $u \in U \subseteq \mathbb{R}^{m_{2}}$ (see [12]).

Theorem 3.3. Consider system (1.2), (1.3), (1.4) under assumptions (A1), (A2), (A3). The function $0 \in$ $L^{\infty}([0,1] ; \mathbb{R})$ is a robust equilibrium point for the system (1.2), (1.3), (1.4) with input $u \in U \subseteq \mathbb{R}^{m_{2}}$, i.e., for all $\varepsilon>0$ and $T \geq 0$ there exists $\delta:=\delta(\varepsilon, T)>0$ such that for every $x_{0} \in L^{\infty}([0,1] ; \mathbb{R}), d \in L^{\infty}([0,+\infty) ; D)$, $u \in L_{\mathrm{loc}}^{\infty}([0,+\infty) ; U)$ with $\sup _{0 \leq z \leq 1}\left|x_{0}(z)\right|+\sup _{t \geq 0}|u(t)|<\delta$ the unique solution $t \rightarrow x_{t} \in L^{\infty}([0,1] ; \mathbb{R})$ of (1.2), (1.3), (1.4) with initial condition $x_{0} \in L^{\infty}([0,1] ; \mathbb{R})$, corresponding to inputs $d \in L^{\infty}([0,+\infty) ; D)$, $u \in L_{\text {loc }}^{\infty}([0,+\infty) ; U)$ satisfies $\sup _{0 \leq z \leq 1}\left|\left(x_{t}\right)(z)\right|<\varepsilon$ for all $t \in[0, T]$.

Proof. The proof is a direct consequence of Theorem 2.2: under assumption (A3) system (3.7) satisfies assumption (H3). Details are left to the reader.

Property 2: Robust global asymptotic stability and Input-to-State stability.

Using the results contained in [12], we are in a position to define the notion of Robust Global Asymptotic Stability and Input-to-State Stability for system (1.2), (1.3), (1.4) under assumptions (A1), (A2), (A3).

Definition 3.4. Consider system (1.2), (1.3), (1.4) under assumptions (A1), (A2), (A3) and assume that for every $x_{0} \in L^{\infty}([0,1] ; \mathbb{R}), d \in L^{\infty}([0,+\infty) ; D), u \in L_{\text {loc }}^{\infty}([0,+\infty) ; U)$, the solution $t \rightarrow x_{t} \in L^{\infty}([0,1] ; \mathbb{R})$ of (1.2), (1.3), (1.4) with initial condition $x_{0} \in L^{\infty}([0,1] ; \mathbb{R})$, corresponding to inputs $d \in L^{\infty}([0,+\infty) ; D)$, $u \in L_{\mathrm{loc}}^{\infty}([0,+\infty) ; U)$ exists for all $t \geq 0$. We say that system (1.2), (1.3), (1.4) is Input-to-State Stable (ISS) from the input $u \in U \subseteq \mathbb{R}^{m_{2}}$ uniformly in $d \in D \subseteq \mathbb{R}^{m_{1}}$, if there exists a continuous non-decreasing function $\gamma: \mathbb{R}_{+} \rightarrow \mathbb{R}_{+}$(called the gain function of the input $u \in L_{\mathrm{loc}}^{\infty}([0,+\infty) ; U)$ ) such that the following properties hold:

Robust Lagrange Stability: For every $\varepsilon>0$ it holds that

$$
\begin{aligned}
& \sup \left\{\sup _{0 \leq z \leq 1}\left|\left(x_{t}\right)(z)\right|-\sup _{0 \leq s \leq t} \gamma(|u(s)|):\right. \\
& \left.t \geq 0, \sup _{0 \leq z \leq 1}\left|x_{0}(z)\right| \leq \varepsilon, d \in L^{\infty}([0,+\infty) ; D), u \in L_{\text {loc }}^{\infty}([0,+\infty) ; U)\right\}<+\infty .
\end{aligned}
$$


Robust Lyapunov Stability: For every $\varepsilon>0$ there exists $\delta:=\delta(\varepsilon)>0$ such that

$$
\begin{aligned}
& \sup \left\{\sup _{0 \leq z \leq 1}\left|\left(x_{t}\right)(z)\right|-\sup _{0 \leq s \leq t} \gamma(|u(s)|):\right. \\
& \left.t \geq 0, \sup _{0 \leq z \leq 1}\left|x_{0}(z)\right| \leq \delta, d \in L^{\infty}([0,+\infty) ; D), u \in L_{\text {loc }}^{\infty}([0,+\infty) ; U)\right\}<\varepsilon .
\end{aligned}
$$

Uniform Robust Attractivity: For every $\varepsilon>0$ and $R \geq 0$ there exists $\tau:=\tau(\varepsilon, R)>0$ such that

$$
\begin{aligned}
& \sup \left\{\sup _{0 \leq z \leq 1}\left|\left(x_{t}\right)(z)\right|-\sup _{0 \leq s \leq t} \gamma(|u(s)|):\right. \\
& \left.t \geq \tau, \sup _{0 \leq z \leq 1}\left|x_{0}(z)\right| \leq R, d \in L^{\infty}([0,+\infty) ; D), u \in L_{\text {loc }}^{\infty}([0,+\infty) ; U)\right\}<\varepsilon .
\end{aligned}
$$

If $U=\{0\}$ then we say that $0 \in L^{\infty}([0,1] ; \mathbb{R})$ is (Uniformly) Robustly Globally Asymptotically Stable (RGAS) for (1.2), (1.3), (1.4).

Lemmas 2.1, Lemma 4.2, Theorem 2.2 in [12] and Theorems 3.1, 3.2, 3.3 give us the following result.

Theorem 3.5. Consider system (1.2), (1.3), (1.4) under assumptions (A1), (A2), (A3). Then the following statements are equivalent:

(a) System (1.2), (1.3), (1.4) is ISS from the input $u \in U \subseteq \mathbb{R}^{m_{2}}$ uniformly in $d \in D \subseteq \mathbb{R}^{m_{1}}$.

(b) There exists $\sigma \in K L$ and a continuous, non-decreasing function $\gamma: \mathbb{R}_{+} \rightarrow \mathbb{R}_{+}$such that for every $x_{0} \in L^{\infty}([0,1] ; \mathbb{R}), d \in L^{\infty}([0,+\infty) ; D), u \in L_{\mathrm{loc}}^{\infty}([0,+\infty) ; U)$, the solution $t \rightarrow x_{t} \in L^{\infty}([0,1] ; \mathbb{R})$ of (1.2), (1.3), (1.4) with initial condition $x_{0} \in L^{\infty}([0,1] ; \mathbb{R})$, corresponding to inputs $d \in L^{\infty}([0,+\infty) ; D)$, $u \in L_{\text {loc }}^{\infty}([0,+\infty) ; U)$ satisfies $\sup _{0 \leq z \leq 1}\left|\left(x_{t}\right)(z)\right| \leq \sigma\left(\sup _{0 \leq z \leq 1}\left|x_{0}(z)\right|, t\right)+\sup _{0 \leq s \leq t} \gamma(|u(s)|)$ for all $t \geq 0$.

(c) System (3.7) is ISS from the input $u \in U \subseteq \mathbb{R}^{m_{2}}$ uniformly in $d \in D \subseteq \mathbb{R}^{m_{1}}$.

Furthermore, if $U=\{0\}$ then the following statement is equivalent to statements (a), (b):

(d) System (1.2), (1.3), (1.4) is Robustly Forward Complete, i.e., for every $T \geq 0$ and $R \geq 0$ it holds that $\sup \left\{\sup _{0 \leq z \leq 1}\left|\left(x_{t}\right)(z)\right|: t \in[0, T], \sup _{0 \leq z \leq 1}\left|x_{0}(z)\right| \leq R, d \in L^{\infty}([0,+\infty) ; D)\right\}<+\infty$, where $x_{t} \in$ $L^{\infty}([0,1] ; \mathbb{R})$ denotes the solution of $(1.2),(1.3)$, (1.4) with initial condition $x_{0} \in L^{\infty}([0,1] ; \mathbb{R})$, corresponding to input $d \in L^{\infty}([0,+\infty) ; D)$, and the property of Uniform Robust Attractivity of Definition 3.3 holds.

Property 3: Lyapunov Characterization of RGAS.

Theorem 3.4 in [12] and the results of Theorems 3.1, 3.2, 3.3, 3.5 allow us to obtain a complete Lyapunov characterization of the RGAS property for system (1.2), (1.3), (1.4).

Theorem 3.6. Consider system (1.2), (1.3), (1.4) under assumptions (A1), (A2), (A3) and assume that $U=$ $\{0\}$. The equilibrium point $0 \in L^{\infty}([0,1] ; \mathbb{R})$ is $R G A S$ for $(1.2),(1.3),(1.4)$ if and only if there exists a functional $V: L^{\infty}([0,1] ; \mathbb{R}) \rightarrow \mathbb{R}_{+}$, a non-decreasing function $Q: \mathbb{R}_{+} \rightarrow \mathbb{R}_{+}$and functions $a_{1}, a_{2} \in K_{\infty}$ such that the following inequalities hold:

$$
\begin{gathered}
a_{1}\left(\sup _{0 \leq z \leq 1}|x(z)|\right) \leq V(x) \leq a_{2}\left(\sup _{0 \leq z \leq 1}|x(z)|\right), \text { for all } x \in L^{\infty}([0,1] ; \mathbb{R}) \\
|V(x)-V(y)| \leq Q\left(\max \left(\sup _{0 \leq z \leq 1}|x(z)|, \sup _{0 \leq z \leq 1}|y(z)|\right)\right) \sup _{0 \leq z \leq 1}|x(z)-y(z)|, \text { for all } x, y \in L^{\infty}([0,1] ; \mathbb{R})
\end{gathered}
$$




$$
V\left(x_{t}\right) \leq \exp (-t) V\left(x_{0}\right), \text { for all } t \geq 0, x_{0} \in L^{\infty}([0,1] ; \mathbb{R}), d \in L^{\infty}([0,+\infty) ; D)
$$

where $x_{t} \in L^{\infty}([0,1] ; \mathbb{R})$ denotes the solution of $(1.2)$, (1.3), (1.4) with initial condition $x_{0} \in L^{\infty}([0,1] ; \mathbb{R})$, corresponding to input $d \in L^{\infty}([0,+\infty) ; D)$.

Again, it should be pointed out that inequality (3.13) guarantees that the functional $V: L^{\infty}([0,1] ; \mathbb{R}) \rightarrow \mathbb{R}_{+}$ is Lipschitz on bounded sets of the state space $L^{\infty}([0,1] ; \mathbb{R})$. However, inequality (3.13) does not guarantee Frechet differentiability of the functional $V: L^{\infty}([0,1] ; \mathbb{R}) \rightarrow \mathbb{R}_{+}$nor that the limit $\lim _{t \rightarrow 0^{+}} t^{-1}\left(V\left(x_{t}\right)-V\left(x_{0}\right)\right)$ exists for the solution $x_{t}$ of $(1.2),(1.3),(1.4)$ with initial condition $x_{0} \in L^{\infty}([0,1] ; \mathbb{R})$, corresponding to input $d \in L^{\infty}([0,+\infty) ; D)$. Notice that inequality (3.14) guarantees that for every $x_{0} \in L^{\infty}([0,1] ; \mathbb{R})$, $d \in L^{\infty}([0,+\infty) ; D)$, the solution $x_{t}$ of $(1.2),(1.3),(1.4)$ with initial condition $x_{0} \in L^{\infty}([0,1] ; \mathbb{R})$, corresponding to input $d \in L^{\infty}([0,+\infty) ; D)$ satisfies $\limsup _{t \rightarrow 0^{+}} t^{-1}\left(V\left(x_{t}\right)-V\left(x_{0}\right)\right) \leq-V\left(x_{0}\right)$.

Property 4: Sufficient conditions for stability properties.

Theorem 3.6 is not the most convenient way of proving RGAS or ISS for (1.2), (1.3), (1.4). For practical purposes we can use the following result, which is a direct consequence of Theorem 2.6 and the equivalent description of system (1.2), (1.3), (1.4) by means of system (3.7). Its proof is omitted.

Theorem 3.7. Consider system (1.2), (1.3), (1.4) under assumptions (H1), (H2), (H3). Assume that there exists a continuous, positive definite and radially unbounded function $W: \mathbb{R}^{N+1} \rightarrow \mathbb{R}_{+}$, a continuous, nondecreasing function $\gamma: \mathbb{R}_{+} \rightarrow \mathbb{R}_{+}$and a constant $\lambda \in(0,1)$ such that the following inequality holds for all $(p, v) \in L^{\infty}\left(\left[-c^{-1}, 0\right) ; \mathbb{R}^{N+1}\right), d \in D, u \in U:$

$$
W(F(d, u, p, v)) \leq \lambda \sup _{-c^{-1} \leq s<0} W(p(s), v(s))+\gamma(|u|)
$$

where $F(d, u, p, v):=\left(F_{1}(d, u, p, v), \ldots, F_{N+1}(d, u, p, v)\right)$ and $F_{i}: D \times U \times L^{\infty}\left(\left[-c^{-1}, 0\right) ; \mathbb{R}^{N+1}\right) \rightarrow \mathbb{R}(i=$ $1, \ldots, N+1)$ are defined by (3.8). Then system (1.2), (1.3), (1.4) is ISS from the input $u \in U \subseteq \mathbb{R}^{m_{2}}$ uniformly in $d \in D \subseteq \mathbb{R}^{m_{1}}$.

\section{Illustrative EXAMPLES}

The first example of this section illustrates the use of Theorem 3.7 for nonlinear uncertain systems described by a single FOH-PDE. Moreover, the fact that a single FOH-PDE can lead to the study of a system described by more than one IDEs is illustrated as well.

Example 4.1. Consider system (1.2), (1.3), (1.4), where $c=1, a(p, z) \equiv 0, N=2, U=\{0\}, K_{1}(d, u, x)=$ $k_{1} \int_{0}^{L}|x(z)| \mathrm{d} z, K_{2}(d, u, x)=k_{2} \int_{0}^{1} x(z) \mathrm{d} z, G(d, u, x)=c d \int_{0}^{1} x(z) \mathrm{d} z, g_{1}(z) \equiv 1, g_{2}(z)=z, k_{1}, k_{2}, k_{3} \in \mathbb{R}_{+}$, $L \in[0,1]$ are constants with $k_{1} L^{2}<2, k_{2} \in\left(0, \frac{6\left(2-k_{1} L^{2}\right)}{k_{1} L^{3}-k_{1} L^{2}+2}\right)$ and $D:=[-1,1] \subset \mathbb{R}$, namely the system

$$
\frac{\partial x}{\partial t}(t, z)+\frac{\partial x}{\partial z}(t, z)=k_{1} \int_{0}^{L}|x(t, z)| \mathrm{d} z+z k_{2} \int_{0}^{1} x(t, z) \mathrm{d} z, x(t, 0)=k_{3} d(t) \int_{0}^{1} x(t, z) \mathrm{d} z,|d(t)| \leq 1 .
$$

For this example, system (3.7) is given by:

$$
\begin{aligned}
& p_{1}(t)=k_{1} \int_{0}^{L}\left|v(t-z)+\int_{t-z}^{t} p_{1}(\tau) \mathrm{d} \tau+\int_{t-z}^{t}(z+\tau-t) p_{2}(\tau) \mathrm{d} \tau\right| \mathrm{d} z \\
& p_{2}(t)=k_{2} \int_{t-1}^{t} v(s) \mathrm{d} s+k_{2} \int_{t-1}^{t}(1+\tau-t) p_{1}(\tau) \mathrm{d} \tau+\frac{k_{2}}{2} \int_{t-1}^{t}(1+\tau-t)^{2} p_{2}(\tau) \mathrm{d} \tau \\
& v(t)=d(t) k_{2} k_{3} \int_{t-1}^{t} v(s) \mathrm{d} s+d(t) k_{2} k_{3} \int_{t-1}^{t}(1+\tau-t) p_{1}(\tau) \mathrm{d} \tau+d(t) \frac{k_{2} k_{3}}{2} \int_{t-1}^{t}(1+\tau-t)^{2} p_{2}(\tau) \mathrm{d} \tau
\end{aligned}
$$


and the solution of (4.1) is related to the solution of (4.2) by means of the equations:

$$
\begin{aligned}
x(t, z) & =v(t-z)+\int_{t-z}^{t} p_{1}(\tau) \mathrm{d} \tau+\int_{t-z}^{t}(z+\tau-t) p_{2}(\tau) \mathrm{d} \tau, \\
p_{1}(t) & =\int_{0}^{L}|x(t, z)| \mathrm{d} z, p_{2}(t)=\int_{0}^{1} x(t, z) \mathrm{d} z .
\end{aligned}
$$

Clearly, assumptions (H1), (H2), (H3) are satisfied for system (4.2) and assumptions (A1), (A2), (A3) are satisfied for system (4.1). In this example we show that the equilibrium point $0 \in L^{\infty}([0,1] ; \mathbb{R})$ is $\operatorname{RGAS}$ for system (4.1) provided that there exists $\gamma>0$ with $\frac{6-k_{2}}{3 k_{2}}>\gamma>\frac{k_{1} L^{3}}{3\left(2-k_{1} L^{2}\right)}$ such that

$$
k_{2} k_{3}<\frac{1}{(3 \gamma+1) \max \left(\frac{k_{2}}{6-(3 \gamma+1) k_{2}}, \frac{k_{1} L}{6 \gamma-(3 \gamma+L) k_{1} L^{2}}\right)+1} .
$$

Indeed, we apply Theorem 3.7 with

$F(d, u, p, v)=\left[\begin{array}{c}k_{1} \int_{-L}^{0}\left|v(s)+\int_{s}^{0} p_{1}(\tau) \mathrm{d} \tau+\int_{s}^{0}(\tau-s) p_{2}(\tau) \mathrm{d} \tau\right| \mathrm{d} s \\ k_{2} \int_{-1}^{0}\left(v(s)+(1+s) p_{1}(s)+\frac{(1+s)^{2}}{2} p_{2}(s)\right) \mathrm{d} s \\ d k_{2} k_{3} \int_{-1}^{0}\left(v(s)+(1+s) p_{1}(s)+\frac{(1+s)^{2}}{2} p_{2}(s)\right) \mathrm{d} s\end{array}\right]$ and $W\left(p_{1}, p_{2}, v\right)=\max \left(\left|p_{1}\right|, \gamma\left|p_{2}\right|, M|v|\right)$, where $M>0$ is a constant that satisfies $\max \left(\frac{6 \gamma k_{2}}{6-(3 \gamma+1) k_{2}}, \frac{6 \gamma k_{1} L}{6 \gamma-(3 \gamma+L) k_{1} L^{2}}\right)<M<6 \gamma \frac{1-k_{2} k_{3}}{k_{2} k_{3}(3 \gamma+1)}$. Using the fact that $|d| \leq 1$, we obtain:

$$
W(F(d, u, p, v)) \leq \max \left(k_{1} L\left(\|v\|+\frac{L}{2}\left\|p_{1}\right\|+\frac{L^{2}}{6}\left\|p_{2}\right\|\right), \max \left(\gamma, M k_{3}\right) k_{2}\left(\|v\|+\frac{1}{2}\left\|p_{1}\right\|+\frac{1}{6}\left\|p_{2}\right\|\right)\right) .
$$

Since $\sup _{-1 \leq s<0} W\left(p_{1}(s), p_{2}(s), v(s)\right)=\max \left(\left\|p_{1}\right\|, \gamma\left\|p_{2}\right\|, M\|v\|\right)$, we get:

$$
(F(d, u, p, v)) \leq \max \left(k_{1} L\left(\frac{1}{M}+\frac{L}{2}+\frac{L^{2}}{6 \gamma}\right), \max \left(\gamma, M k_{3}\right) k_{2}\left(\frac{1}{M}+\frac{1}{2}+\frac{1}{6 \gamma}\right)\right) \sup _{-1 \leq s<0} W\left(p_{1}(s), p_{2}(s), v(s)\right) .
$$

Consequently, (3.15) holds with $\lambda:=\max \left(k_{1} L\left(\frac{1}{M}+\frac{L}{2}+\frac{L^{2}}{6 \gamma}\right), \max \left(\gamma, M k_{3}\right) k_{2}\left(\frac{1}{M}+\frac{1}{2}+\frac{1}{6 \gamma}\right)\right)<1($ and $\gamma \equiv 0)$.

The second example of this section shows how the results of the previous sections can be used for control purposes. The example deals with a linear FOH-PDE and helps us to illustrate many points arising in control problems in a pedagogical way.

Example 4.2. Consider systems (1.2), (1.3), (1.4), where $c=1, a(p, z) \equiv 0, N=1, U=\mathbb{R}, K_{1}(u, x)=x(1)$, $g_{1}(z)=g, g \in \mathbb{R}$ is a constant and $d \in D$ is irrelevant. This problem was studied in [14] and a boundary feedback control of the form:

$$
G(u, x)=\int_{0}^{1} k(z) x(z) \mathrm{d} z
$$

was designed, where the kernel $k \in C^{1}([0,1] ; \mathbb{R})$ was explicitly given and guaranteed finite time stability for the corresponding closed-loop system. Here we will provide an alternative methodology for the design of a robust stabilizer for system (1.2), (1.3), (1.4) with $G(u, x)=u \in \mathbb{R}$, namely, the system

$$
\frac{\partial x}{\partial t}(t, z)+\frac{\partial x}{\partial z}(t, z)=g \limsup _{h \rightarrow 0^{+}}\left(h^{-1} \int_{1-h}^{1} x(t, z) \mathrm{d} z\right), \quad x(t, 0)=u(t) .
$$


Notice that we have replaced the functional $K_{1}(u, x)=x(1)$ by the functional $K_{1}(u, x)=$ $\limsup _{h \rightarrow 0^{+}}\left(h^{-1} \int_{1-h}^{1} x(z) \mathrm{d} z\right)$ : the two functionals coincide when $x \in L^{\infty}([0,1] ; \mathbb{R})$ is continuous at $z=1$. However, the functional $K_{1}(u, x)=\limsup _{h \rightarrow 0^{+}}\left(h^{-1} \int_{1-h}^{1} x(z) \mathrm{d} z\right)$ is well-defined for all $x \in L^{\infty}([0,1] ; \mathbb{R})$ and both functionals $G(u, x)=u \in \mathbb{R}$ and $K_{1}(u, x)=\limsup _{h \rightarrow 0^{+}}\left(h^{-1} \int_{1-h}^{1} x(z) \mathrm{d} z\right)$ satisfy assumption (A1). For this example, system (3.7) is given by:

$$
\begin{aligned}
& p(t)=g \int_{-1}^{0} p(t+s) \mathrm{d} s+\limsup _{h \rightarrow 0^{+}}\left(h^{-1} \int_{1-h}^{1} v(t-z) \mathrm{d} z\right) \\
& v(t)=u(t)
\end{aligned}
$$

and the solution of (4.6) is related to the solution of (4.7) by means of the equations:

$$
x(t, z)=v(t-z)+g \int_{t-z}^{t} p(w) \mathrm{d} w, p(t)=\limsup _{h \rightarrow 0^{+}}\left(h^{-1} \int_{1-h}^{1} x(t, z) \mathrm{d} z\right) .
$$

However, notice that since $v_{t} \in L^{\infty}([-1,0) ; \mathbb{R})$, it holds that

$\limsup _{h \rightarrow 0^{+}}\left(h^{-1} \int_{1-h}^{1} v(t-z) \mathrm{d} z\right)=\limsup _{h \rightarrow 0^{+}}\left(h^{-1} \int_{t-1}^{t-1+h} v(s) \mathrm{d} s\right)=v(t-1)$ for $t \geq 0$ a.e.. Therefore, for this example, system (3.7) is given by:

$$
\begin{aligned}
& p(t)=g \int_{-1}^{0} p(t+s) \mathrm{d} s+v(t-1) \\
& v(t)=u(t) .
\end{aligned}
$$

Equations (4.9) imply that the following differential equation holds for almost all $t \geq 0$ for which the solution of (4.9) exists:

$$
\frac{\mathrm{d}}{\mathrm{d} t}(p(t)-v(t-1))=g(p(t)-v(t-1))-g p(t-1)+g \mathrm{v}(t-1) .
$$

Consequently, the following equation holds for all $t \geq t_{0} \geq 0$ for which the solution of (4.9) exists:

$p(t)=v(t-1)+\exp \left(g\left(t-t_{0}\right)\right)\left(p\left(t_{0}\right)-v\left(t_{0}-1\right)\right)-g \int_{t_{0}}^{t} \exp (g(t-s)) p(s-1) \mathrm{d} s+g \int_{t_{0}}^{t} \exp (g(t-s)) v(s-1) \mathrm{d} s$.

Using (4.9) and (4.11) with $t_{0}=t-1$ we conclude that system (4.9) satisfies the following equations for $t \geq 1$ a.e. (provided that the solution exists):

$$
\begin{aligned}
p(t) & =v(t-1)+g \int_{t-2}^{t-1}(\exp (g)-\exp (g(t-s-1))) p(s) \mathrm{d} s+g \int_{t-2}^{t-1} \exp (g(t-s-1)) v(s) \mathrm{d} s . \\
v(t) & =u(t)
\end{aligned}
$$

It is clear that the feedback law:

$$
u(t)=a p(t)-g \int_{t-1}^{t}(\exp (g)-\exp (g(t-s))) p(s) \mathrm{d} s-g \int_{t-1}^{t} \exp (g(t-s)) v(s) \mathrm{d} s
$$

with $|a|<1$ guarantees that the closed-loop system (4.9) with (4.13) satisfies for $t \geq 1$ a.e. (provided that the solution exists):

$$
\begin{aligned}
& p(t)=a p(t-1) \\
& v(t)=a p(t)-g \int_{-1}^{0}(\exp (-g w) v(t+w)+(\exp (g)-\exp (-g w)) p(t+w)) \mathrm{d} w
\end{aligned}
$$


Clearly, assumptions (H1), (H2), (H3) are satisfied for the closed-loop system (4.9) with (4.13). Theorem 2.2 guarantees the local existence of a solution of the closed-loop system (4.9) with (4.13) for every initial condition. Using (4.11) with $t_{0}=0$, we are in a position to show that there exists a constant $G>0$ such that the estimate $|p(t)| \leq G\left(\sup _{-1 \leq s<0}(|p(s)|)+\sup _{-1 \leq s<0}(|v(s)|)\right)$ holds for every initial condition and for all $t \in[0,1)$ for which the solution exists. Exploiting the previous estimate for $p(t)$ and applying the GronwallBellman Lemma to equation $v(t)=a p(t)-g \int_{-1}^{0}(\exp (-g w) v(t+w)+(\exp (g)-\exp (-g w)) p(t+w)) \mathrm{d} w$ for the continuous function $v(t)-a p(t)$ we are in a position to show that there exists a constant $N>0$ such that the estimate $|v(t)| \leq N\left(\sup _{-1<s<0}(|p(s)|)+\sup _{-1<s<0}(|v(s)|)\right)$ holds for every initial condition and for all $t \in[0,1)$ for which the solution exists. Using Theorem 2.2 and a standard contradiction argument we conclude that the solution of the closed-loop system (4.9) with (4.13) exists for all $t \in[0,1)$ and for every initial condition (because otherwise the solution should be unbounded). Using (4.9) (and more specifically, the equation $\left.v(t)=p(t+1)-g \int_{-1}^{0} p(t+1+s) \mathrm{d} s\right)$ we can conclude from (4.14) that $p(t)=a^{[t]} p(t-[t])$ and $v(t)=a^{[t]}\left(a p(t-[t])-g a \int_{[t+1]}^{t+1} p(w-[w]) \mathrm{d} w-g \int_{t}^{[t+1]} p(w-[w]) \mathrm{d} w\right)$ for $t \geq 1$ a.e., where $[t]$ denotes the integer part of $t \geq 1$. At this point, we should emphasize that we have achieved exponential stabilization of system (4.9) by means of the feedback law (4.13). Indeed, there exists a constant $M \geq 1$ so that the following estimate holds for the solution of the closed-loop system (4.9) with (4.13):

$$
\sup _{t-1 \leq s<t}|v(s)|+\sup _{t-1 \leq s<t}|p(s)| \leq M \sigma_{a}(t)\left(\sup _{-1 \leq s<0}|v(s)|+\sup _{-1 \leq s<0}|p(s)|\right) \text {, for all } t \geq 0
$$

where

$$
\sigma_{a}(t)=\left\{\begin{array}{cl}
1 \quad \text { if } & t \in[0,2] \\
|a|^{[t-1]} & \text { if } \quad t>2 .
\end{array}\right.
$$

More specifically, estimate (4.15) and definition (4.16) show that for $a=0$ we have achieved finite-time stabilization of the system (4.9) by means of the feedback law (4.13). However, we have not achieved stabilization of system (4.6) by means of a state feedback law (in terms of the state of system (4.6)). This remains to be shown. Indeed, using (4.8) we get from (4.13):

$$
\begin{aligned}
& u(t)=a p(t)-g \int_{t-1}^{t}(\exp (g)-\exp (g(t-s))) p(s) \mathrm{d} s-g \int_{t-1}^{t} \exp (g(t-s)) v(s) \mathrm{d} s \\
& =a p(t)-g \int_{t-1}^{t}(\exp (g)-\exp (g(t-s))) p(s) \mathrm{d} s-g \int_{0}^{1} \exp (g z) v(t-z) \mathrm{d} z \\
& =a p(t)-g \int_{t-1}^{t}(\exp (g)-\exp (g(t-s))) p(s) \mathrm{d} s+g^{2} \int_{0}^{1} \exp (g z) \int_{t-z}^{t} p(s) \mathrm{d} s \mathrm{~d} z-g \int_{0}^{1} \exp (g z) x(t, z) \mathrm{d} z .
\end{aligned}
$$

Integrating by parts we get:

$$
\begin{aligned}
& g^{2} \int_{0}^{1} \exp (g z) \int_{t-z}^{t} p(s) \mathrm{d} s \mathrm{~d} z=g \int_{0}^{1} \frac{\mathrm{d}}{\mathrm{d} z}(\exp (g z)) \int_{t-z}^{t} p(s) \mathrm{d} s \mathrm{~d} z \\
& =g \int_{0}^{1} \frac{\mathrm{d}}{\mathrm{d} z}\left(\exp (g z) \int_{t-z}^{t} p(s) \mathrm{d} s\right) \mathrm{d} z-g \int_{0}^{1} \exp (g z) \frac{\mathrm{d}}{\mathrm{d} z}\left(\int_{t-z}^{t} p(s) \mathrm{d} s\right) \mathrm{d} z \\
& =g \exp (g) \int_{t-1}^{t} p(s) \mathrm{d} s-g \int_{0}^{1} \exp (g z) p(t-z) \mathrm{d} z=g \int_{t-1}^{t}(\exp (g)-\exp (g(t-s))) p(s) \mathrm{d} s
\end{aligned}
$$

and consequently we obtain

$$
u(t)=a \limsup _{h \rightarrow 0^{+}}\left(h^{-1} \int_{1-h}^{1} x(t, z) \mathrm{d} z\right)-g \int_{0}^{1} \exp (g z) x(t, z) \mathrm{d} z
$$

which is a boundary feedback law of the form (4.5) with $k(z)=-g \exp (g z)$ for $a=0$. Notice that the closedloop system (4.6) with (4.17) satisfies assumptions (A1), (A2), (A3). An estimate which displays exponential 
convergence can be derived for the solution of the closed-loop system (4.6) with (4.17). Indeed, using (4.8) we get $\sup _{0<z \leq 1}|x(t, z)| \leq \sup _{t-1 \leq s<t}|v(s)|+|g| \sup _{t-1 \leq s<t}|p(s)|$. The previous inequality, estimate (4.15) and the fact that (4.8) holds, allow us (by selecting $p(s) \equiv 0$ for $s \in[-1,0)$, which by virtue of $(4.8)$ gives $v(-q)=x(0, q)$ for $q \in(0,1])$ to obtain the estimate:

$$
\sup _{0<z \leq 1}|x(t, z)| \leq(1+|g|) M \sigma_{a}(t) \sup _{0<z \leq 1}|x(0, z)|, \text { for all } t \geq 0
$$

where $\sigma_{a}$ is defined in (4.16). More specifically, estimate (4.18) allows us to conclude that the feedback law (4.5) with $k(z)=-g \exp (g z)$ achieves finite-time stabilization of system (4.6).

The analysis presented so far allowed us to obtain some different features from the analysis in [14]:

1. The implementation of the feedback law: It follows from (4.8) that the feedback law (4.17) can be implemented by using the following distributed delay feedback law:

$$
u(t)=a x(t, 1)-g \int_{t-1}^{t}(\exp (g)-\exp (g(t-s))) x(s, 1) \mathrm{d} s-g \int_{t-1}^{t} \exp (g(t-s)) x(s, 0) \mathrm{d} s
$$

for all $t \geq 0$ for which $x_{t} \in C^{0}([0,1] ; \mathbb{R})$. The feedback law (4.19) shows that we do not need to measure the whole state profile when $x_{t} \in C^{0}([0,1] ; \mathbb{R})$ : we only need to measure $x(t, z)$ at two different space points $z=0$ and $z=1$.

2. The issue of the state space: It should be emphasized that the feedback law (4.17) for $a=0$, is exactly the same with that obtained in [14]. However, we have proved that estimate (4.18) holds for initial conditions $x(0, z)=x_{0}(z)$ for $z \in[0,1]$ with $x_{0} \in L^{\infty}([0,1] ; \mathbb{R})$. Moreover, no compatibility condition (e.g., $\left.\lim _{z \rightarrow 0^{+}} x_{0}(z)=-g \int_{0}^{1} \exp (g z) x_{0}(z) \mathrm{d} z\right)$ is required to hold.

3. Control actuator errors: The implementation of the feedback law (4.17) for $a=0$, may result to the equation:

$$
u(t)=-g \int_{0}^{1} \exp (g z) x(t, z) \mathrm{d} z+w(t), \text { for } t \geq 0
$$

where $w \in L^{\infty}\left(\mathbb{R}_{+} ; \mathbb{R}\right)$ is the control actuator error. Using the framework described in the present work, we are in a position to study the closed-loop system (4.6) with (4.20). In this case, we get the integral delay system:

$$
\begin{aligned}
& p(t)=v(t-1)+g \int_{-1}^{0} p(t+s) \mathrm{d} s \\
& v(t)=w(t)-g \int_{-1}^{0}(\exp (g)-\exp (-g s)) p(t+s) \mathrm{d} s-g \int_{-1}^{0} \exp (-g s) v(t+s) \mathrm{d} s
\end{aligned}
$$

with $w \in L^{\infty}\left(\mathbb{R}_{+} ; \mathbb{R}\right)$ as input. Using (4.12), we get for all $t \geq 1$ :

$$
p(t)=w(t-1), \quad v(t)=w(t)-g \int_{-1}^{0} w(t+s) \mathrm{d} s
$$

which implies (by means of a similar analysis to that of the disturbance-free case) the existence of constants $M \geq 1, \gamma \geq 0$ such that the following estimate holds:

$$
\sup _{t-1 \leq s<t}|v(s)|+\sup _{t-1 \leq s<t}|p(s)| \leq M \sigma_{0}(t)\left(\sup _{-1 \leq s<0}|v(s)|+\sup _{-1 \leq s<0}|p(s)|\right)+\gamma \sup _{0 \leq s \leq t}|w(s)| \text {, for all } t \geq 0
$$

where $\sigma_{0}(t)$ is defined in (4.16) with $a=0$. Estimate (4.23) shows that the ISS property holds for system (4.21). Estimate (4.23) shows (using exactly the same arguments as above) that the following estimate 
holds for the solution of (4.6) with (4.20):

$$
\sup _{0<z \leq 1}|x(t, z)| \leq(1+|g|) M \sigma_{0}(t) \sup _{0<z \leq 1}|x(0, z)|+(1+|g|) \gamma \sup _{0 \leq s \leq t}|w(s)| \text {, for all } t \geq 0
$$

which is exactly the ISS property for the closed-loop system (4.6) with (4.20).

Having presented the analysis of the example, we are in a position to point out certain shortcomings of the proposed methodology of the conversion of a single FOH-PDE to a system described by IDEs:

i) The major shortcoming of the above analysis is that the researcher studies a different system (an integral delay system) from the original one (a system described by first-order hyperbolic pdes). This is important, because a stabilizing feedback law for (4.9) may not be equivalent to a state feedback law for (4.6): it may involve delay terms. Therefore, a stabilizing feedback law for (4.9), when expressed in the original "coordinates" may not give us a closed-loop system of the form (4.6) but rather a system described by a single first-order hyperbolic PDE with delays.

ii) Another shortcoming of the above analysis is the specific form (1.2), (1.3), (1.4) for which the conversion to a system described by IDEs can be used. Not all systems described by first-order hyperbolic pdes can be expressed by $(1.2),(1.3),(1.4)$ : the requirement that the functionals $K_{i}: W \times L^{\infty}([0,1] ; \mathbb{R}) \rightarrow \mathbb{R}$ $(i=1, \ldots, N)$ are not allowed to depend on $z \in[0,1]$ is restrictive. There are systems which are studied in [14] but cannot be studied in the proposed framework. However, one must bear in mind that a preliminary integral transformation can be applied so that the transformed system is of the form (1.2), (1.3), (1.4).

The following example is a nonlinear extension of the previous example. The example illustrates that the proposed approach is not limited to linear control systems. Instead, we can study highly nonlinear control systems described by FOH-PDEs.

Example 4.3. Consider system (1.2), (1.3), (1.4), where $c=1, a(p, z) \equiv 0, N=1, U=\mathbb{R}, K_{1}(u, x)=$ $\varphi\left(\limsup _{h \rightarrow 0^{+}}\left(h^{-1} \int_{1-h}^{1} x(z) \mathrm{d} z\right)\right)$, where $\varphi: \mathbb{R} \rightarrow \mathbb{R}$ is a globally Lipschitz function with $\varphi(0)=0, G(u, x)=u \in$ $\mathbb{R}, g_{1}(z) \equiv 1$ and $d \in D$ is irrelevant, namely, the system

$$
\frac{\partial x}{\partial t}(t, z)+\frac{\partial x}{\partial z}(t, z)=\varphi\left(\limsup _{h \rightarrow 0^{+}}\left(h^{-1} \int_{1-h}^{1} x(t, z) \mathrm{d} z\right)\right), x(t, 0)=u(t) .
$$

Notice again that we have replaced the functional $K_{1}(u, x)=\varphi(x(1))$ by the functional $K_{1}(u, x)=$ $\varphi\left(\limsup _{h \rightarrow 0^{+}}\left(h^{-1} \int_{1-h}^{1} x(z) \mathrm{d} z\right)\right)$ : the two functionals coincide when $x \in L^{\infty}([0,1] ; \mathbb{R})$ is continuous at $z=1$.

However, the functional $K_{1}(u, x)=\varphi\left(\limsup _{h \rightarrow 0^{+}}\left(h^{-1} \int_{1-h}^{1} x(z) \mathrm{d} z\right)\right)$ is well-defined for all $x \in L^{\infty}([0,1] ; \mathbb{R})$ and both functionals $G(u, x)=u \in \mathbb{R}$ and $K_{1}(u, x)=\varphi\left(\limsup _{h \rightarrow 0^{+}}\left(h^{-1} \int_{1-h}^{1} x(z) \mathrm{d} z\right)\right)$ satisfy assumption (A1). For this example, system (3.7) is given by:

$$
\begin{aligned}
& p(t)=\varphi\left(\int_{-1}^{0} p(t+s) \mathrm{d} s+\limsup _{h \rightarrow 0^{+}}\left(h^{-1} \int_{1-h}^{1} v(t-z) \mathrm{d} z\right)\right) \\
& v(t)=u(t)
\end{aligned}
$$

and the solution of (4.25) is related to the solution of (4.26) by means of the equations:

$$
x(t, z)=v(t-z)+\int_{t-z}^{t} p(w) \mathrm{d} w, p(t)=\varphi\left(\limsup _{h \rightarrow 0^{+}}\left(h^{-1} \int_{1-h}^{1} x(t, z) \mathrm{d} z\right)\right) .
$$


Since $v_{t} \in L^{\infty}([-1,0) ; \mathbb{R})$, it holds that $\limsup _{h \rightarrow 0^{+}}\left(h^{-1} \int_{1-h}^{1} v(t-z) \mathrm{d} z\right)=\limsup _{h \rightarrow 0^{+}}\left(h^{-1} \int_{t-1}^{t-1+h} v(s) \mathrm{d} s\right)=v(t-1)$ for $t \geq 0$ a.e.. Therefore, for this example, system (3.7) is given by:

$$
\begin{aligned}
& p(t)=\varphi\left(\int_{t-1}^{t} p(s) \mathrm{d} s+v(t-1)\right) \\
& v(t)=u(t) .
\end{aligned}
$$

A feedback stabilizer for (4.28) can be designed by using successive approximations for the solution map of (4.28). Notice that for $t \geq 1$ we get from (4.28) $\int_{t-1}^{t} p(s) \mathrm{d} s=\int_{t-1}^{t} \varphi\left(v(s-1)+\int_{s-1}^{s} p(w) \mathrm{d} w\right) \mathrm{d} s$. A first approximation of the integral $\int_{t-1}^{t} p(s) \mathrm{d} s$ can be the functional $\int_{t-1}^{t} \varphi\left(v(s-1)+\int_{s-1}^{t-1} p(w) \mathrm{d} w\right) \mathrm{d} s$ with approximation error for $t \geq 1$ :

$$
\begin{aligned}
& \left|\int_{t-1}^{t} p(s) \mathrm{d} s-\int_{t-1}^{t} \varphi\left(v(s-1)+\int_{s-1}^{t-1} p(w) \mathrm{d} w\right) \mathrm{d} s\right| \\
& =\left|\int_{t-1}^{t} \varphi\left(v(s-1)+\int_{s-1}^{s} p(w) \mathrm{d} w\right) \mathrm{d} s-\int_{t-1}^{t} \varphi\left(v(s-1)+\int_{s-1}^{t-1} p(w) \mathrm{d} w\right) \mathrm{d} s\right| \\
& \leq \int_{t-1}^{t}\left|\varphi\left(v(s-1)+\int_{s-1}^{s} p(w) \mathrm{d} w\right)-\varphi\left(v(s-1)+\int_{s-1}^{t-1} p(w) \mathrm{d} w\right)\right| \mathrm{d} s \\
& \leq L \int_{t-1}^{t}\left|\int_{t-1}^{s} p(w) \mathrm{d} w\right| \mathrm{d} s \leq \frac{L}{2} \sup _{t-1 \leq s<t}(|p(s)|)
\end{aligned}
$$

where $L \geq 0$ is the global Lipschitz constant of the function $\varphi: \mathbb{R} \rightarrow \mathbb{R}$. Therefore, the following feedback law is suggested:

$$
u(t)=-\int_{t-1}^{t} \varphi\left(v(s)+\int_{s}^{t} p(w) \mathrm{d} w\right) \mathrm{d} s
$$

Clearly, assumptions (H1), (H2), (H3) are satisfied for the closed-loop system (4.28) with (4.29). We show next that $0 \in L^{\infty}\left([-1,0) ; \mathbb{R}^{2}\right)$ is GAS for the closed-loop system (4.28) with (4.29) provided that

$$
L<\sqrt{2} .
$$

Using (4.27), this implies that $0 \in L^{\infty}([0,1] ; \mathbb{R})$ is GAS for the closed-loop system (4.25) with

$$
u(t)=-\int_{0}^{1} \varphi(x(t, z)) \mathrm{d} z
$$

Notice that assumptions (A1), (A2), (A3) hold the closed-loop system (4.25) with (4.31). Theorem 2.2 guarantees local existence of the solution of the closed-loop system (4.28) with (4.29) for every initial condition. Using the estimate $|\varphi(x)| \leq L|x|$ for all $x \in \mathbb{R}$ (which holds by virtue of the fact that $\varphi(0)=0$ and that $L \geq 0$ is the global Lipschitz constant of the function $\varphi: \mathbb{R} \rightarrow \mathbb{R}$ ), we apply the Gronwall-Bellman Lemma to the measurable functions $p(t), v(t)$ and obtain the existence of a constant $G \geq 0$ such that the estimate $|p(t)|+|v(t)| \leq G\left(\sup _{-1 \leq s<0}(|p(s)|)+\sup _{-1 \leq s<0}(|v(s)|)\right)$ holds for every initial condition and for almost all $t \in[0,1)$ for which the solution exists. Using Theorem 2.2 and a standard contradiction argument we conclude that the solution of the closed-loop system (4.28) with (4.29) exists for all $t \in[0,1)$ and for every initial condition (because otherwise the solution should be unbounded). Next, we apply Theorem 2.6 to the system:

$$
\begin{aligned}
& p(t)=\varphi\left(\int_{t-1}^{t} \varphi\left(v(s-1)+\int_{s-1}^{s} p(w) \mathrm{d} w\right) \mathrm{d} s-\int_{t-1}^{t} \varphi\left(v(s-1)+\int_{s-1}^{t-1} p(w) \mathrm{d} w\right) \mathrm{d} s\right) \\
& v(t)=-\int_{t-1}^{t} \varphi\left(\int_{s-1}^{t-1} \varphi\left(v(w)+\int_{w}^{w+1} p(\tau) \mathrm{d} \tau\right) \mathrm{d} w-\int_{s-1}^{s} \varphi\left(v(w)+\int_{w}^{s} p(\tau) \mathrm{d} \tau\right) \mathrm{d} w\right) \mathrm{d} s
\end{aligned}
$$


which describes the evolution of the solution of the closed-loop system (4.28) with (4.29) for $t \geq 1$. Applying Theorem 2.6 to system (4.32) with $W(p, v):=\max (|p|, M|v|)$, where $M>0$ satisfies $2 L^{2} M<3\left(2-L^{2}\right)$, we get:

$$
W(F(p, v)) \leq \max \left(\frac{L^{2}}{2}\|p\|, M \frac{L^{2}}{3}\|p\|+M \frac{L^{2}}{2}\|v\|\right)
$$

where $F(p, v)=\left[\begin{array}{c}\varphi\left(\int_{-1}^{0} \varphi\left(v(s-1)+\int_{s-1}^{s} p(w) \mathrm{d} w\right) \mathrm{d} s-\int_{-1}^{0} \varphi\left(v(s-1)+\int_{s-1}^{-1} p(w) \mathrm{d} w\right) \mathrm{d} s\right) \\ -\int_{-1}^{0} \varphi\left(\int_{s-1}^{-1} \varphi\left(v(w)+\int_{w}^{w+1} p(\tau) \mathrm{d} \tau\right) \mathrm{d} w-\int_{s-1}^{s} \varphi\left(v(w)+\int_{w}^{s} p(\tau) \mathrm{d} \tau\right) \mathrm{d} w\right) \mathrm{d} s\end{array}\right]$.

Inequality (4.33) is obtained by repeated use of the Lipschirz inequality for $\varphi: \mathbb{R} \rightarrow \mathbb{R}$ and the fact that $|\varphi(x)| \leq L|x|$ for all $x \in \mathbb{R}$. Since $\sup _{-1 \leq s<0} W(p(s), v(s))=\max (\|p\|, M\|v\|)$, we get:

$$
W(F(p, v)) \leq \max \left(\frac{L^{2}}{2}, M \frac{L^{2}}{3}+\frac{L^{2}}{2}\right) \sup _{-1 \leq s<0} W(p(s), v(s))
$$

Consequently, (2.24) holds with $\lambda:=\max \left(\frac{L^{2}}{2}, M \frac{L^{2}}{3}+\frac{L^{2}}{2}\right)$ (and $\left.\tilde{\gamma} \equiv 0\right)$. Since

- $0 \in L^{\infty}\left([-1,0) ; \mathbb{R}^{2}\right)$ is GAS for system (4.32);

- the solution of the closed-loop system (4.28) with (4.29) satisfies (4.32) for $t \geq 1$;

- there exists a constant $G \geq 0$ such that the estimate $|p(t)|+|v(t)| \leq G\left(\sup _{-1 \leq s<0}(|p(s)|)+\sup _{-1 \leq s<0}(|v(s)|)\right)$ holds for every initial condition and for almost all $t \in[0,1)$.

It follows that statement (c) of Theorem 2.4 holds. Hence, $0 \in L^{\infty}\left([-1,0) ; \mathbb{R}^{2}\right)$ is GAS for the closed-loop system (4.28) with (4.29).

\section{Concluding Remarks}

This paper establishes the equivalence between systems described by a single first-order hyperbolic partial differential equation and systems described by integral delay equations. System-theoretic results are provided for both classes of systems:

1. the Boundedness-Implies-Continuation property and the property of Lipschitz dependence on initial conditions (Thms. 2.1, 3.1 and 3.2);

2. the robustness of an equilibrium point for systems with external inputs (Thms. 2.2 and 3.3);

3. characterizations of the Input-to-State Stability and Robust Global Asymptotic Stability (Thms. 2.4 and 3.5);

4. converse Lyapunov results (Thms. 2.5 and 3.6)

5. sufficient conditions for Input-to-State Stability and Robust Global Asymptotic Stability (Thms. 2.6 and 3.7).

The proposed framework can allow the study of discontinuous solutions for nonlinear systems described by a single first-order hyperbolic partial differential equation under the effect of measurable inputs acting on the boundary and/or on the differential equation. This aspect is important from a control-theoretic point of view because all systems are subject to disturbances (modelling errors, control actuator errors and measurement errors), which are typically modeled by measurable inputs. Illustrative examples have showed that the conversion of a system described by a single first-order hyperbolic partial differential equation to an integral delay system can simplify considerably the solution of the corresponding robust feedback stabilization problem and the stability analysis. 


\section{Appendix. Proofs of Theorems 3.1 And 3.2}

Proof of Theorem 3.1. The proof is divided in three steps.

Step 1. We show that system (3.7) satisfies assumptions (H1), (H2). Therefore, by virtue of Theorem 2.1 for every input $w \in L_{\text {loc }}^{\infty}([0,+\infty) ; W)$ and for every initial condition $(p, v) \in L^{\infty}\left(\left[-c^{-1}, 0\right) ; \mathbb{R}^{N+1}\right)$ that satisfies $(3.4)$ for certain $x_{0} \in L^{\infty}([0,1] ; \mathbb{R})$, system $(3.7)$ admits a unique solution. The obtained solution of system (3.7) gives us directly (by means of $(3.4)$ and $(3.5))$ a locally bounded mapping $\left[0, t_{\max }\right)$ э $t \rightarrow x_{t} \in L^{\infty}([0,1] ; \mathbb{R})$ satisfying $(1.3),(1.4),(3.3)$ for $t \in\left[0, t_{\max }\right)$ a.e. with $(p, v) \in L_{\mathrm{loc}}^{\infty}\left(\left[0, t_{\max }\right) ; \mathbb{R}^{N+1}\right)$, and $(3.9)$ for all $t \in\left[0, t_{\max }\right)$ and almost all $z \in[0,1]$.

Step 2. We show that for every $w \in L_{\text {loc }}^{\infty}([0,+\infty) ; W), x_{0} \in L^{\infty}([0,1] ; \mathbb{R})$ there exists at most one locally bounded mapping $\left[0, t_{\max }\right)$ э $t \rightarrow x_{t} \in L^{\infty}([0,1] ; \mathbb{R})$ satisfying (1.3), (1.4), (3.3) for $t \in\left[0, t_{\max }\right)$ a.e. with $(p, v) \in L_{\mathrm{loc}}^{\infty}\left(\left[0, t_{\max }\right) ; \mathbb{R}^{N+1}\right)$, and $(3.9)$ for all $t \in\left[0, t_{\max }\right)$ and almost all $z \in[0,1]$. Therefore, the mapping obtained in Step 1 is the unique mapping that satisfies (1.3), (1.4), (3.3) for $t \in\left[0, t_{\max }\right)$ a.e. with $(p, v) \in$ $L_{\text {loc }}^{\infty}\left(\left[0, t_{\max }\right) ; \mathbb{R}^{N+1}\right)$, and $(3.9)$ for all $t \in\left[0, t_{\max }\right)$ and almost all $z \in[0,1]$. This means that for every initial condition $(p, v) \in L^{\infty}\left(\left[-c^{-1}, 0\right) ; \mathbb{R}^{N+1}\right)$ that satisfies (3.4) for the given $x_{0} \in L^{\infty}([0,1] ; \mathbb{R})$ the solution of system (3.7) will be the same.

Step 3. Making use of the results of Theorem 2.1, we show that if $t_{\max }<+\infty$ then lim sup $\sup \left|\left(x_{t}\right)(z)\right|=+\infty$. $t \rightarrow t_{\max }^{-} 0 \leq z \leq 1$

Step 1: Assumption (A2) guarantees that assumption (H2) holds for system (3.7). Likewise, the validity of assumption (H1) for system (3.7) is a direct consequence of assumption (A1). To see this, let $R \geq 0,(p, v) \in$ $L^{\infty}\left(\left[-c^{-1}, 0\right) ; \mathbb{R}^{N+1}\right),(\tilde{p}, \tilde{v}) \in L^{\infty}\left(\left[-c^{-1}, 0\right) ; \mathbb{R}^{N+1}\right)$ with $\|(p, v)\| \leq R,\|(\tilde{p}, \tilde{v})\| \leq R$ be given and let $x, \tilde{x} \in$ $L^{\infty}((0,1] ; \mathbb{R})$ be defined by $x=A(p) v+B(p), \tilde{x}=A(\tilde{p}) \tilde{v}+B(\tilde{p})$, where $A(p): L^{\infty}\left(\left[-c^{-1}, 0\right) ; \mathbb{R}\right) \rightarrow L^{\infty}((0,1] ; \mathbb{R})$ and $B: L^{\infty}\left(\left[-c^{-1}, 0\right) ; \mathbb{R}^{N}\right) \rightarrow L^{\infty}((0,1] ; \mathbb{R})$ are the mappings defined by $(3.6 \mathrm{~b})$, i.e.,

$$
\begin{aligned}
& x(z)=\exp \left(\int_{-c^{-1} z}^{0} a(p(s), z+c s) \mathrm{d} s\right) v\left(-c^{-1} z\right)+\int_{-c^{-1} z}^{0} \exp \left(\int_{\tau}^{0} a(p(s), z+c s) \mathrm{d} s\right) g(z+c \tau) p(\tau) \mathrm{d} \tau \\
& \text { for } z \in[0,1] a . e . \\
& \tilde{x}(z)=\exp \left(\int_{-c^{-1} z}^{0} a(\tilde{p}(s), z+c s) \mathrm{d} s\right) \tilde{v}\left(-c^{-1} z\right)+\int_{-c^{-1} z}^{0} \exp \left(\int_{\tau}^{0} a(\tilde{p}(s), z+c s) \mathrm{d} s\right) g(z+c \tau) \tilde{p}(\tau) \mathrm{d} \tau, \\
& \text { for } z \in[0,1] \text { a.e. }
\end{aligned}
$$

Define:

$$
\tilde{M}(R):=\max _{z \in[0,1],|p| \leq R}|a(p, z)| \text { and } C:=\max _{z \in[0,1]}|g(z)|
$$

and notice that (A.1) and definitions (A.3) imply:

$$
\max \left(\sup _{0 \leq z \leq 1}|x(z)|, \sup _{0 \leq z \leq 1}|\tilde{x}(z)|\right) \leq\left(1+c^{-1} C\right) \exp \left(c^{-1} \tilde{M}(R)\right) R .
$$

Using (3.2), definitions (3.8) and (A.4), we obtain:

$$
\begin{aligned}
& |F(w, p, v)| \leq \sigma\left(\left(1+c^{-1} C\right) \exp \left(c^{-1} \tilde{M}(R)\right) R\right), \\
& \text { for all }(w, p, v) \in W \times L^{\infty}\left(\left[-c^{-1}, 0\right) ; \mathbb{R}^{N+1}\right) \text { with }\|(p, v)\| \leq R \text { and }|w| \leq \mathbb{R} .
\end{aligned}
$$

Inequality (A.5) shows that inequality (2.2) holds for the non-decreasing function

$a(R):=\sigma\left(\left(1+c^{-1} C\right) \exp \left(c^{-1} \tilde{M}(R)\right) R\right)$ for all $R \geq 0$ (notice that definition (A.3) implies that $\tilde{M}(R)$ is non-decreasing). 
Using (3.1), definitions (3.8) and (A.4), we obtain:

$$
|F(w, p, v)-F(w, \tilde{p}, \tilde{v})| \leq L(K(R)) h \sup _{0<z \leq h}(|x(z)-\tilde{x}(z)|)+L(K(R)) \sup _{h<z \leq 1}(|x(z)-\tilde{x}(z)|),
$$
for all $h \in(0,1), w \in W$ with $|w| \leq R$

where $K(R):=\left(1+c^{-1} C\right) \exp \left(c^{-1} \tilde{M}(R)\right) R$.

Define $L_{a}(R):=\sup \left\{\frac{|a(p, z)-a(\tilde{p}, z)|}{|p-\tilde{p}|}: p \neq \tilde{p},|p| \leq R,|\tilde{p}| \leq R, z \in[0,1]\right\}$ and notice that $L_{a}(R)<+\infty$ for all $R \geq 0$ (since $a \in C^{0}\left(\mathbb{R}^{N} \times[0,1] ; \mathbb{R}\right)$ is assumed to be locally Lipschitz). Using (A.1), (A.2) and definitions (A.3) we obtain for $z \in[0,1]$ a.e.:

$$
|x(z)-\tilde{x}(z)| \leq B_{1}(R)\left|v\left(-c^{-1} z\right)-\tilde{v}\left(-c^{-1} z\right)\right|+B_{2}(R) \int_{-c^{-1} z}^{0}|p(s)-\tilde{p}(s)| \mathrm{d} s
$$

where $B_{1}(R):=\exp \left(\tilde{M}(R) c^{-1}\right), B_{2}(R):=\left(R L_{a}(R)+C+C R c^{-1} L_{a}(R)\right) \exp \left(3 \tilde{M}(R) c^{-1}\right)$. Combining (A.6) and (A.7) we obtain for all $h \in(0,1), w \in W$ with $|w| \leq R$ :

$$
\begin{aligned}
& |F(w, p, v)-F(w, \tilde{p}, \tilde{v})| \leq L(K(R)) B_{1}(R) h \sup _{-c^{-1} h \leq s<0}(|v(s)-\tilde{v}(s)|) \\
& +2 L(K(R)) B_{2}(R) c^{-1} h \sup _{-c^{-1} h \leq s<0}|p(s)-\tilde{p}(s)| \\
& +L(K(R)) B_{1}(R) \sup _{c^{-1} \leq s<-c^{-1} h}(|v(s)-\tilde{v}(s)|) \\
& +L(K(R)) B_{2}(R) c^{-1} \sup _{c^{-1} \leq s<-c^{-1} h}|p(s)-\tilde{p}(s)| .
\end{aligned}
$$

Inequality (A.8) shows that inequality (2.1) holds for appropriate non-decreasing functions $M: \mathbb{R}_{+} \rightarrow \mathbb{R}_{+}$, $N: \mathbb{R}_{+} \rightarrow \mathbb{R}_{+}$.

Let arbitrary $x_{0} \in L^{\infty}([0,1] ; \mathbb{R})$ and $w \in L_{\mathrm{loc}}^{\infty}([0,+\infty) ; W)$. Consider now the solution of system (3.7) corresponding to input $w \in L_{\mathrm{loc}}^{\infty}([0,+\infty) ; W)$ with initial condition $(p, v) \in L^{\infty}\left(\left[-c^{-1}, 0\right) ; \mathbb{R}^{N+1}\right)$ with $p(s) \equiv 0$ and (3.4), i.e.,

$$
v(t)=\exp \left(-\int_{t}^{0} a(0, c(s-t)) \mathrm{d} s\right) x_{0}(-c t), \text { for almost all } t \in\left[-c^{-1}, 0\right) .
$$

Theorem 2.1 guarantees that there exists $t_{\max }:=t_{\max }\left(x_{0}, w\right) \in(0,+\infty]$ so that system (3.7) admits a unique solution $(p, v) \in L_{\text {loc }}^{\infty}\left(\left[-c^{-1}, t_{\text {max }}\right) ; \mathbb{R}^{N+1}\right)$. Using (3.5) and (A.9), we obtain a locally bounded mapping $\left[0, t_{\max }\right)$ э $t \rightarrow x_{t} \in L^{\infty}([0,1] ; \mathbb{R})$, which satisfies (1.3), (1.4), (3.3) for $t \in\left[0, t_{\max }\right)$ a.e. and (3.9) for all $t \in\left[0, t_{\max }\right)$ and almost all $z \in[0,1]$.

Step 2: We show that by contradiction that for every $w \in L_{\text {loc }}^{\infty}([0,+\infty) ; W), x_{0} \in L^{\infty}([0,1] ; \mathbb{R})$ there exists at most one locally bounded mapping $\left[0, t_{\max }\right)$ э $t \rightarrow x_{t} \in L^{\infty}([0,1] ; \mathbb{R})$ satisfying $(1.3),(1.4),(3.3)$ for $t \in\left[0, t_{\max }\right)$ a.e. with $(p, v) \in L_{\mathrm{loc}}^{\infty}\left(\left[0, t_{\max }\right) ; \mathbb{R}^{N+1}\right)$, and $(3.9)$ for all $t \in\left[0, t_{\max }\right)$ and almost all $z \in[0,1]$.

Suppose that there exist $w \in L_{\text {loc }}^{\infty}([0,+\infty) ; W), x_{0} \in L^{\infty}([0,1] ; \mathbb{R})$, a constant $\delta>0$ and two locally bounded mappings $[0, \delta) \ni t \rightarrow x_{t} \in L^{\infty}([0,1] ; \mathbb{R}),[0, \delta) \ni t \rightarrow \tilde{x}_{t} \in L^{\infty}([0,1] ; \mathbb{R})$ so that $(3.9)$ holds for all $t \in[0, \delta)$ and almost all $z \in[0,1]$, equation

$$
\begin{aligned}
\tilde{x}(t, z)= & \int_{\max \left(0, t-c^{-1} z\right)}^{t} \exp \left(\int_{\tau}^{t} a(\tilde{p}(s), z+c(s-t)) \mathrm{d} s\right) g(z+c(\tau-t)) \tilde{p}(\tau) \mathrm{d} \tau \\
& +\exp \left(\int_{\max \left(0, t-c^{-1} z\right)}^{t} a(\tilde{p}(s), z+c(s-t)) \mathrm{d} s\right)\left\{\begin{array}{ccc}
\tilde{v}\left(t-c^{-1} z\right) & \text { for } \quad z \leq c t \\
x_{0}(z-c t) & \text { for } \quad z>c t
\end{array}\right.
\end{aligned}
$$


for all $t \in[0, \delta)$ and almost all $z \in[0,1],(1.3),(1.4),(3.3)$ hold for $t \in[0, \delta)$ a.e., equations

$$
\begin{gathered}
\tilde{p}_{i}(t)=K_{i}\left(w(t), \tilde{x}_{t}\right), \text { for } i=1, \ldots, N \text { and } \tilde{p}(t)=\left(\tilde{p}_{1}(t), \ldots, \tilde{p}_{N}(t)\right)^{\prime} \in \mathbb{R}^{N} \\
\tilde{v}(t)=G\left(w(t), \tilde{x}_{t}\right)
\end{gathered}
$$

hold for $t \in[0, \delta)$ a.e. and such that

$$
\sup _{t \in[0, \delta)} \sup _{z \in[0,1]}\left|\left(x_{t}\right)(z)-\left(\tilde{x}_{t}\right)(z)\right|>0
$$

Let $t_{1} \in[0, \delta)$ be the greatest lower bound of all times $t \in[0, \delta)$ for which $\sup _{z \in[0,1]}\left|\left(x_{t}\right)(z)-\left(\tilde{x}_{t}\right)(z)\right|>0$. Notice that by virtue of (A.13), $t_{1} \in[0, \delta)$ is well defined and that $\sup _{t \in\left[0, t_{1}\right)} \sup _{z \in[0,1]}\left|\left(x_{t}\right)(z)-\left(\tilde{x}_{t}\right)(z)\right|=0$ for the case that $t_{1}>0$.

We show next that there exists $h>0$ so that $\sup _{t \in\left[0, t_{1}+h\right)} \sup _{z \in[0,1]}\left|\left(x_{t}\right)(z)-\left(\tilde{x}_{t}\right)(z)\right|=0$, which contradicts the fact that $t_{1} \in[0, \delta)$ is the greatest lower bound of all times $t \in[0, \delta)$ for which $\sup _{z \in[0,1]}\left|\left(x_{t}\right)(z)-\left(\tilde{x}_{t}\right)(z)\right|>0$.

An important property that is used at this point is the so-called classical semigroup property (see [11]), i.e., the fact that every locally bounded mapping $\left[0, t_{\max }\right) \ni t \rightarrow x_{t} \in L^{\infty}([0,1] ; \mathbb{R})$ satisfying (1.3), (1.4), (3.3) for $t \in\left[0, t_{\max }\right)$ a.e. with $(p, v) \in L_{\mathrm{loc}}^{\infty}\left(\left[0, t_{\max }\right) ; \mathbb{R}^{N+1}\right)$, and $(3.9)$ for all $t \in\left[0, t_{\max }\right)$ and almost all $z \in[0,1]$ satisfies the following identity for all $t \in\left[0, t_{\max }\right)$ and for all $t_{0} \in[0, t]$ :

$$
\begin{aligned}
& x(t, z)=\int_{\max \left(0, t-t_{0}-c^{-1} z\right)}^{t-t_{0}} \exp \left(\int_{\tau}^{t-t_{0}} a\left(p\left(t_{0}+s\right), z+c\left(s+t_{0}-t\right)\right) \mathrm{d} s\right) g\left(z+c\left(\tau+t_{0}-t\right)\right) p\left(t_{0}+\tau\right) \mathrm{d} \tau \\
& +\exp \left(\int_{\max \left(0, t-t_{0}-c^{-1} z\right)}^{t-t_{0}} a\left(p\left(t_{0}+s\right), z+c\left(s+t_{0}-t\right)\right) \mathrm{d} s\right)\left\{\begin{array}{c}
v\left(t-c^{-1} z\right) \quad \text { for } z+c t_{0} \leq c t \\
\left(x_{t_{0}}\right)\left(z+c t_{0}-c t\right) \quad \text { for } z+c t_{0}>c t
\end{array},\right.
\end{aligned}
$$

for $z \in[0,1]$ a.e.

Therefore, identity (A.14) holds for all $t \in[0, \delta)$ and for all $t_{0} \in[0, t]$. Similarly, the following identity

$$
\begin{aligned}
& \tilde{x}(t, z)=\int_{\max \left(0, t-t_{0}-c^{-1} z\right)}^{t-t_{0}} \exp \left(\int_{\tau}^{t-t_{0}} a\left(\tilde{p}\left(t_{0}+s\right), z+c\left(s+t_{0}-t\right)\right) \mathrm{d} s\right) g\left(z+c\left(\tau+t_{0}-t\right)\right) \tilde{p}\left(t_{0}+\tau\right) \mathrm{d} \tau \\
& +\exp \left(\int_{\max \left(0, t-t_{0}-c^{-1} z\right)}^{t-t_{0}} a\left(\tilde{p}\left(t_{0}+s\right), z+c\left(s+t_{0}-t\right)\right) \mathrm{d} s\right)\left\{\begin{array}{c}
\tilde{v}\left(t-c^{-1} z\right) \quad \text { for } z+c t_{0} \leq c t \\
\left(\tilde{x}_{t_{0}}\right)\left(z+c t_{0}-c t\right) \quad \text { for } z+c t_{0}>c t
\end{array}\right.
\end{aligned}
$$

for $z \in[0,1]$ a.e.

holds for all $t \in[0, \delta)$ and for all $t_{0} \in[0, t]$. Using (A.14) and (A.15) with $t_{0} \in\left(0, t_{1}\right)$ for the case $t_{1}>0$ and $t_{0}=0$ for the case $t_{1}=0$ in conjunction with (1.3), (3.3), (A.3), (A.11), (A.12) (which implies that $p(s)=\tilde{p}(s)$, $v(s)=\tilde{v}(s), x_{s}=\tilde{x}_{s}$ for all $\left.s \in\left[0, t_{0}\right]\right)$, we obtain:

$$
\begin{aligned}
& |x(t, z)-\tilde{x}(t, z)| \leq \\
& \left(t-t_{0}\right) \exp \left(3 \tilde{M}(R)\left(t-t_{0}\right)\right)\left(C+C R L_{a}(R)\left(t-t_{0}\right)+2 R L_{a}(R)\right) \sup _{t_{0} \leq s \leq t}|p(s)-\tilde{p}(s)| \\
& +\exp \left(\tilde{M}(R)\left(t-t_{0}\right)\right)\left\{\begin{array}{c}
\left|v\left(t-c^{-1} z\right)-\tilde{v}\left(t-c^{-1} z\right)\right| \quad \text { for } z+c t_{0} \leq c t \\
0 \text { for } z+c t_{0}>c t
\end{array}\right.
\end{aligned}
$$


for $z \in[0,1]$ a.e. and for all $t \in\left[t_{0}, \frac{t_{1}+\delta}{2}\right]$, where

$$
\begin{aligned}
& R=\sup _{0 \leq s \leq \frac{t_{1}+\delta}{2}} \max \left(|p(s)|,|\tilde{p}(s)|,|v(s)|,|\tilde{v}(s)|, \sup _{0 \leq z \leq 1}\left|\left(x_{s}\right)(z)\right|, \sup _{0 \leq z \leq 1}\left|\left(\tilde{x}_{s}\right)(z)\right|,|w(s)|\right) \\
& L_{a}(R):=\sup \left\{\frac{|a(p, z)-a(\tilde{p}, z)|}{|p-\tilde{p}|}: p \neq \tilde{p},|p| \leq R,|\tilde{p}| \leq R, z \in[0,1]\right\} .
\end{aligned}
$$

Using (1.3), (3.1), (3.3), (A.11) and (A.12) we obtain:

$$
\begin{gathered}
|p(s)-\tilde{p}(s)| \leq 2 L(R) \sup _{0 \leq z \leq 1}|x(s, z)-\tilde{x}(s, z)| \text {, for almost all } s \in\left[t_{0}, \frac{t_{1}+\delta}{2}\right] \\
\left|v\left(t-c^{-1} z\right)-\tilde{v}\left(t-c^{-1} z\right)\right| \leq L(R)\left(t-c^{-1} z-t_{0}\right) \sup _{0 \leq s \leq t-c^{-1} z-t_{0}}\left|x\left(t-c^{-1} z, s\right)-\tilde{x}\left(t-c^{-1} z, s\right)\right| \\
+L(R) \sup _{t-c^{-1} z-t_{0}<s \leq 1}\left|x\left(t-c^{-1} z, s\right)-\tilde{x}\left(t-c^{-1} z, s\right)\right| \\
\text { for almost all } t \in\left(t_{0}, \min \left(\frac{t_{1}+\delta}{2}, t_{0}+c^{-1}\right)\right) \text { and } 0 \leq z<c\left(t-t_{0}\right) .
\end{gathered}
$$

We define for all $h \in\left(0, \min \left(\frac{\delta-t_{1}}{2}, c^{-1}\right)\right)$ :

$$
\Delta(h):=\sup _{t_{0}<t \leq t_{0}+h}\left(\sup _{c\left(t-t_{0}\right)<z \leq 1}|x(t, z)-\tilde{x}(t, z)|\right), E(h):=\sup _{t_{0}<t \leq t_{0}+h}\left(\sup _{0 \leq z \leq c\left(t-t_{0}\right)}|x(t, z)-\tilde{x}(t, z)|\right) .
$$

Using (A.16), (A.17), (A.18) and definitions (A.19) we obtain for all $h \in\left(0, \min \left(\frac{\delta-t_{1}}{2}, c^{-1}\right)\right)$ :

$$
\begin{gathered}
\Delta(h) \leq h \Gamma_{1}(R) \max (\Delta(h), E(h)) \\
E(h) \leq h \Gamma_{1}(R) \max (\Delta(h), E(h))+h \Gamma_{2}(R) E(h)+\Gamma_{2}(R) \Delta(h)
\end{gathered}
$$

where $\Gamma_{1}(R):=\exp \left(3 M(R) c^{-1}\right)\left(C+C R L_{a}(R) c^{-1}+2 R L_{a}(R)\right) 2 L(R), \Gamma_{2}(R):=\exp \left(M(R) c^{-1}\right) L(R)$. Using (A.21) and assuming that $h \leq \frac{1}{2\left(\Gamma_{1}(R)+\Gamma_{2}(R)\right)}$ we get $E(h) \leq \max \left(1,2 \Gamma_{2}(R)\right) \Delta(h)$. In this case, inequality (A.20) implies $\Delta(h) \leq h \Gamma_{1}(R) \max \left(1,2 \Gamma_{2}(R)\right) \Delta(h)$ which gives:

$$
\Delta(h)=E(h)=0, \text { for all } h \in\left(0, \frac{1}{2} \min \left(c^{-1}, \frac{\delta-t_{1}}{2}, \frac{1}{2\left(\Gamma_{1}(R)+\Gamma_{2}(R)\right)\left(1+2 \Gamma_{2}(R)\right)}\right)\right] .
$$

Implication (A.22), definitions (A.19) and the fact that $\sup _{t \in\left[0, t_{0}\right]} \sup _{z \in[0,1]}\left|\left(x_{t}\right)(z)-\left(\tilde{x}_{t}\right)(z)\right|=0$ imply that $\sup _{t \in\left[0, t_{0}+h\right]} \sup _{z \in[0,1]}\left|\left(x_{t}\right)(z)-\left(\tilde{x}_{t}\right)(z)\right|=0$.

Since $t_{0} \in\left(0, t_{1}\right]$ is arbitrarily close to $t_{1}$, we get $\sup _{t \in\left[0, t_{1}+h\right)} \sup _{z \in[0,1]}\left|\left(x_{t}\right)(z)-\left(\tilde{x}_{t}\right)(z)\right|=0$.

Thus, the locally bounded mapping $\left[0, t_{\max }\right)$ э $t \rightarrow x_{t} \in L^{\infty}([0,1] ; \mathbb{R})$, which satisfies (1.3), (1.4), (3.3) for $t \in\left[0, t_{\max }\right)$ a.e. and $(3.9)$ for all $t \in\left[0, t_{\max }\right)$ and almost all $z \in[0,1]$, which was found in Step 1 is unique.

Step 3: In this step we show that if $t_{\max }<+\infty$ then $\limsup _{t \rightarrow t^{-}} \sup _{0<z<1}\left|\left(x_{t}\right)(z)\right|=+\infty$ and that there exist nondecreasing functions $P: \mathbb{R}_{+} \rightarrow \mathbb{R}_{+}, Q: \mathbb{R}_{+} \rightarrow \mathbb{R}_{+}$such that for every $x_{0} \in L^{\infty}([0,1] ; \mathbb{R}), y_{0} \in L^{\infty}([0,1] ; \mathbb{R})$, $w \in L_{\text {loc }}^{\infty}([0,+\infty) ; W)$ estimate $(3.10)$ holds.

Indeed, if $t_{\max }<+\infty$ for the unique solution $(p, v) \in L_{\mathrm{loc}}^{\infty}\left(\left[-c^{-1}, t_{\max }\right) ; \mathbb{R}^{N+1}\right)$ of $(3.7)$ corresponding to input $w \in L_{\mathrm{loc}}^{\infty}([0,+\infty) ; W)$ with initial condition $(p, v) \in L^{\infty}\left(\left[-c^{-1}, 0\right) ; \mathbb{R}^{N+1}\right)$ satisfying $p(s) \equiv 0$ and (A.9), then 
Theorem 2.1 guarantees that $\lim \sup \left\|\left(p_{t}, v_{t}\right)\right\|=+\infty$. By virtue of inequality (3.2) and equations (1.3), (3.3), $\limsup \left\|\left(p_{t}, v_{t}\right)\right\|=+\infty$ cannot happen unless limsup $\sup \left|\left(x_{t}\right)(z)\right|=+\infty$. The proof is complete. $t \rightarrow t_{\max }^{-}$

Proof of Theorem 3.2. Theorem 2.1 guarantees that there exist non-decreasing functions $\tilde{P}: \mathbb{R}_{+} \rightarrow \mathbb{R}_{+}, \tilde{G}:$ $\mathbb{R}_{+} \rightarrow \mathbb{R}_{+}$such that for every $v_{0} \in L^{\infty}\left(\left[-c^{-1}, 0\right) ; \mathbb{R}\right), \tilde{v}_{0} \in L^{\infty}\left(\left[-c^{-1}, 0\right) ; \mathbb{R}\right), w \in L_{\text {loc }}^{\infty}([0,+\infty) ; W)$ it holds that:

$$
\left\|p_{t}-\tilde{p}_{t}\right\|+\left\|v_{t}-\tilde{v}_{t}\right\| \leq \tilde{G}(s(t)) \exp (\tilde{P}(s(t)) t)\left\|v_{0}-\tilde{v}_{0}\right\|, \text { for all } t \in[0, \delta)
$$

where $\delta=\min \left(t_{\max }\left(v_{0}, w\right), t_{\max }\left(\tilde{v}_{0}, w\right)\right), s(t):=\max \left(\sup _{0 \leq \tau \leq t}\left\|\left(p_{\tau}, v_{\tau}\right)\right\|, \sup _{0 \leq \tau \leq t}\left\|\left(\tilde{p}_{\tau}, \tilde{v}_{\tau}\right)\right\|, \sup _{0 \leq \tau \leq t}\|w(\tau)\|\right)$ and $(p, v) \in L_{\text {loc }}^{\infty}\left(\left[-c^{-1}, t_{\max }\left(v_{0}, w\right)\right) ; \mathbb{R}^{N+1}\right),(\tilde{p}, \tilde{v}) \in L_{\text {loc }}^{\infty}\left(\left[-c^{-1}, t_{\max }\left(\tilde{v}_{0}, w\right)\right) ; \mathbb{R}^{N+1}\right)$ are the unique solutions of $(3.7)$ corresponding to input $w \in L_{\text {loc }}^{\infty}([0,+\infty) ; W)$ with initial conditions $\left(0, v_{0}\right) \in L^{\infty}\left(\left[-c^{-1}, 0\right) ; \mathbb{R}^{N+1}\right)$ and $\left(0, \tilde{v}_{0}\right) \in L^{\infty}\left(\left[-c^{-1}, 0\right) ; \mathbb{R}^{N+1}\right)$, respectively.

Let $x_{0} \in L^{\infty}([0,1] ; \mathbb{R}), \tilde{x}_{0} \in L^{\infty}([0,1] ; \mathbb{R}), w \in L_{\mathrm{loc}}^{\infty}([0,+\infty) ; W)$ and consider the locally bounded mappings $\left[0, t_{\max }\left(x_{0}, w\right)\right)$ э $t \rightarrow x_{t} \in L^{\infty}([0,1] ; \mathbb{R}),\left[0, t_{\max }\left(\tilde{x}_{0}, w\right)\right)$ э $t \rightarrow \tilde{x}_{t} \in L^{\infty}([0,1] ; \mathbb{R})$, which satisfy $(1.3),(1.4),(3.3)$ for $t \in\left[0, t_{\max }\right)$ a.e. and (3.9) for all $t \in\left[0, t_{\max }\right)$ and almost all $z \in[0,1]$ as well as the analogous equations with $\tilde{x}$ replacing $x$ and $\tilde{x}_{0}$ replacing $x_{0}$. The mappings $\left[0, t_{\max }\left(x_{0}, w\right)\right)$ э $t \rightarrow x_{t} \in L^{\infty}([0,1] ; \mathbb{R}),\left[0, t_{\max }\left(\tilde{x}_{0}, w\right)\right)$ э $t \rightarrow \tilde{x}_{t} \in L^{\infty}([0,1] ; \mathbb{R})$ are found by obtaining the solutions $(p, v) \in L_{\mathrm{loc}}^{\infty}\left(\left[-c^{-1}, t_{\max }\left(v_{0}, w\right)\right) ; \mathbb{R}^{N+1}\right)$, $(\tilde{p}, \tilde{v}) \in L_{\mathrm{loc}}^{\infty}\left(\left[-c^{-1}, t_{\mathrm{max}}\left(\tilde{v}_{0}, w\right)\right) ; \mathbb{R}^{N+1}\right)$ of $(3.7)$ with input $w \in L_{\mathrm{loc}}^{\infty}([0,+\infty) ; W)$ and initial conditions $\left(0, v_{0}\right) \in L^{\infty}\left(\left[-c^{-1}, 0\right) ; \mathbb{R}^{N+1}\right),\left(0, \tilde{v}_{0}\right) \in L^{\infty}\left(\left[-c^{-1}, 0\right) ; \mathbb{R}^{N+1}\right)$, respectively, and by using (3.5), (A.9) and

$$
\tilde{v}(t)=\exp \left(-\int_{t}^{0} a(0, c(s-t)) \mathrm{d} s\right) \tilde{x}_{0}(-c t), \text { for almost all } t \in\left[-c^{-1}, 0\right) .
$$

Inequality (3.10) for appropriate non-decreasing functions $P: \mathbb{R}_{+} \rightarrow \mathbb{R}_{+}, Q: \mathbb{R}_{+} \rightarrow \mathbb{R}_{+}$is a direct consequence of (A.16) with $t_{0}=0,($ A.23), (A.9), (A.24), (1.3), (3.3) and (3.2).

In order to show that the mapping $t \rightarrow x_{t} \in L^{\mu}([0,1] ; \mathbb{R})$ is continuous on $\left[0, t_{\max }\right)$ for every $\mu \in[1,+\infty)$, it suffices to use the classical semigroup property (identity (A.14)) and to show that for every $\mu \in[1,+\infty), \varepsilon>0$ there exists $\tilde{\delta}>0$ such that

$$
\int_{0}^{1}\left|x(t, z)-x_{0}(z)\right|^{\mu} \mathrm{d} z \leq \varepsilon^{\mu}, \text { for all } t \in[0, \tilde{\delta}] .
$$

Since $C^{0}([0,1] ; \mathbb{R})$ is dense in $L^{\mu}([0,1] ; \mathbb{R})$ for every $\mu \in[1,+\infty)$ for every $\varepsilon>0$ and $x_{0} \in L^{\infty}([0,1] ; \mathbb{R})$ there exists $\tilde{x}_{0} \in C^{0}([0,1] ; \mathbb{R})$ with $\int_{0}^{1}\left|\tilde{x}_{0}(z)-x_{0}(z)\right|^{\mu} \mathrm{d} z \leq(\varepsilon / 3)^{\mu}$ and $\sup _{0 \leq z \leq 1}\left|\tilde{x}_{0}(z)\right| \leq \sup _{0 \leq z \leq 1}\left|x_{0}(z)\right|$. Moreover, there exists $\delta \in(0,1)$ such that $\sup _{0 \leq s \leq \delta \leq \delta \leq 1} \sup _{s \leq z \leq 1}\left|\tilde{x}_{0}(z-s)-\tilde{x}_{0}(z)\right| \leq \frac{\varepsilon}{3}$. Using the inequality

$$
\begin{aligned}
\left(\int_{\delta}^{1}\left|x_{0}(z-s)-x_{0}(z)\right|^{\mu} \mathrm{d} z\right)^{1 / \mu} \leq & \left(\int_{s}^{1}\left|x_{0}(z-s)-\tilde{x}_{0}(z-s)\right|^{\mu} \mathrm{d} z\right)^{1 / \mu} \\
& +\left(\int_{s}^{1}\left|\tilde{x}_{0}(z-s)-\tilde{x}_{0}(z)\right|^{\mu} \mathrm{d} z\right)^{1 / \mu}+\left(\int_{s}^{1}\left|\tilde{x}_{0}(z)-x_{0}(z)\right|^{\mu} \mathrm{d} z\right)^{1 / \mu}
\end{aligned}
$$

we obtain $\int_{\delta}^{1}\left|x_{0}(z-s)-x_{0}(z)\right|^{\mu} \mathrm{d} z \leq \varepsilon^{\mu}$ for all $s \in[0, \delta]$. Consequently, for every $\mu \in[1,+\infty)$, for every $\varepsilon>0$ and $x_{0} \in L^{\infty}([0,1] ;)$ there exists $\delta \in(0,1)$ such that $\sup _{0 \leq s \leq \delta} \int_{s}^{1}\left|x_{0}(z-s)-x_{0}(z)\right|^{\mu} \mathrm{d} z \leq \varepsilon^{\mu}$.

Using the identity $\int_{0}^{1}\left|x(t, z)-x_{0}(z)\right|^{\mu} \mathrm{d} z=\int_{0}^{c t}\left|x(t, z)-x_{0}(z)\right|^{\mu} \mathrm{d} z+\int_{c t}^{1}\left|x(t, z)-x_{0}(z)\right|^{\mu} \mathrm{d} z$ for all $t \in$ $\left[0, c^{-1}\right]$, we get 


$$
\int_{0}^{1}\left|x(t, z)-x_{0}(z)\right|^{\mu} \mathrm{d} z \leq 2^{\mu} c R^{\mu} t+\int_{c t}^{1}\left|x(t, z)-x_{0}(z)\right|^{\mu} \mathrm{d} z, \text { for all } t \in\left[0, \min \left(c^{-1} / 2, t_{\max } / 2\right)\right]
$$

where $R=\sup _{0 \leq s \leq t_{\max } / 2} \max \left(|p(s)|,|v(s)|, \sup _{0 \leq z \leq 1}\left|\left(x_{s}\right)(z)\right|,|w(s)|\right)$. Moreover, using (3.9) and definitions (A.3) we get for all $t \in\left[0, \min \left(c^{-1} / 2, t_{\max } / 2\right)\right]$ and almost all $z \in(c t, 1]$ :

$$
\left|x(t, z)-x_{0}(z)\right| \leq \exp (\tilde{M}(R) t)(C+\tilde{M}(R)) R t+\left|x_{0}(z-c t)-x_{0}(z)\right| .
$$

Combining (A.26) and (A.27) we obtain for all $t \in\left[0, \min \left(c^{-1} / 2, t_{\max } / 2\right)\right]$ :

$$
\int_{0}^{1}\left|x(t, z)-x_{0}(z)\right|^{\mu} \mathrm{d} z \leq 2^{\mu} c R^{\mu} t+2^{\mu} \exp (\mu \tilde{M}(R) t)(C+\tilde{M}(R))^{\mu} R^{\mu} t^{\mu}+2^{\mu} \int_{c t}^{1}\left|x_{0}(z-c t)-x_{0}(z)\right|^{\mu} \mathrm{d} z
$$

Let $\delta \in(0,1)$ be such that $2^{\mu} \sup _{0 \leq s \leq \delta} \int_{s}^{1}\left|x_{0}(z-s)-x_{0}(z)\right|^{\mu} \mathrm{d} z \leq \frac{1}{3} \varepsilon^{\mu}$. It follows from the previous inequality in conjunction with (A.28) that (A.25) holds with $\tilde{\delta}=\min \left(\frac{1}{2 c}, \frac{t_{\max }}{2}, \frac{\delta}{c}, \frac{\varepsilon}{3(2 R)^{\mu} c}, \frac{\exp \left(-\tilde{M}(R) c^{-1}\right)}{2 R(C+\tilde{M}(R))}\left(\frac{\varepsilon}{3}\right)^{1 / \mu}\right)$. The proof is complete.

\section{REFERENCES}

[1] O. Aamo, Disturbance Rejection in $2 \times 2$ Linear Hyperbolic Systems. IEEE Trans. Autom. Control 58 (2013) $1095-1106$.

[2] G. Bastin and J.-M. Coron, Further Results on Boundary Feedback Stabilization of $2 \times 2$ Hyperbolic Systems Over a Bounded Interval. In Proc. of IFAC Nolcos 2010, Bologna, Italy (2010).

[3] G. Bastin and J.-M. Coron, On Boundary Feedback Stabilization of Non-Uniform Linear $2 \times 2$ Hyperbolic Systems Over a Bounded Interval. Syst. Control Lett. 60 (2011) 900-906.

[4] K.L. Cooke and D.W. Krumme, Differential-Difference Equations and Nonlinear Initial-Boundary Value Problems for Linear Hyperbolic Partial Differential Equations. J. Math. Anal. Appl. 24 (1968) 372-387.

[5] J.-M. Coron, G. Bastin and B. d'Andrea-Novel, Dissipative Boundary Conditions for One-Dimensional Nonlinear Hyperbolic Systems. SIAM J. Control Optim. 47 (2008) 1460-1498.

[6] J.-M. Coron, R. Vazquez, M. Krstic, and G. Bastin, Local Exponential H2 Stabilization of a $2 \times 2$ Quasilinear Hyperbolic System Using Backstepping. SIAM J. Control Optim. 51 (2013) 2005-2035.

[7] A. Diagne, G. Bastin and J.-M. Coron, Lyapunov Exponential Stability of 1-d Linear Hyperbolic Systems of Balance Laws. Automatica 48 (2012) 109-114.

[8] A.V. Fillipov, Differential Equations with Discontinuous Right-Hand Sides. Kluwer Academic Publishers, Dordrecht (1988).

[9] S.-Y. Ha and A. Tzavaras, Lyapunov Functionals and $\mathrm{L}^{1}$-Stability for Discrete Velocity Boltzmann Equations. Commun. Math. Phys. 239 (2003) 65-92.

[10] J.K. Hale and S.M.V. Lunel, Introduction to Functional Differential Equations. Springer-Verlag, New York (1993).

[11] I. Karafyllis, P. Pepe and Z.-P. Jiang, Stability Results for Systems Described by Coupled Retarded Functional Differential Equations and Functional Difference Equations. Nonlinear Anal., Theory Methods Appl. 71 (2009) 3339-3362.

[12] I. Karafyllis and Z.-P. Jiang, Stability and Stabilization of Nonlinear Systems. Commun. Control Eng. Springer-Verlag London (2011).

[13] I. Karafyllis and M. Krstic, Nonlinear Stabilization under Sampled and Delayed Measurements, and with Inputs Subject to Delay and Zero-Order Hold. IEEE Trans. Autom. Control 57 (2012) 1141-1154.

[14] M. Krstic and A. Smyshlyaev, Backstepping Boundary Control for First-Order Hyperbolic PDEs and Application to Systems With Actuator and Sensor Delays. Syst. Control Lett. 57 (2008) 750-758.

[15] M. Krstic, Delay Compensation for Nonlinear, Adaptive, and PDE Systems. Birkhuser Boston (2009).

[16] M. Krstic, Input Delay Compensation for Forward Complete and Strict-Feedforward Nonlinear Systems. IEEE Trans. Autom. Control 55 (2010) 287-303.

[17] T.T. Li, Controllability and Observability for Quasilinear Hyperbolic Systems, vol. 3. Higher Education Press, Beijing (2009).

[18] D. Melchor-Aguilar, V. Kharitonov and R. Lozano, Stability Conditions for Integral Delay Systems. Int. J. Robust Nonlinear Control $2020101-15$.

[19] D. Melchor-Aguilar, On Stability of Integral Delay Systems. Appl. Math. Comput. 217 (2010) 3578-3584.

[20] D. Melchor-Aguilar, Exponential Stability of Some Linear Continuous Time Difference Systems. Syst. Control Lett. 61 (2012) $62-68$. 
[21] L. Pavel and L. Chang, Lyapunov-Based Boundary Control for a Class of Hyperbolic Lotka-Volterra Systems. IEEE Trans. Autom. Control 57 (2012) 701-714.

[22] P. Pepe, The Lyapunov's Second Method for Continuous Time Difference Equations. Int. J. Robust Nonlinear Control 13 (2003) 1389-1405.

[23] C. Prieur and F. Mazenc, ISS-Lyapunov Functions for Time-Varying Hyperbolic Systems of Balance Laws. Math. Control, Signals Syst. 24 (2012) 111-134.

[24] C. Prieur, J. Winkin and G. Bastin, Robust Boundary Control of Systems of Conservation Laws. Math. Control Signals Syst. 20 (2008) 173-197.

[25] V. Rasvan and S.I. Niculescu, Oscillations in Lossless Propagation Models: a Liapunov-Krasovskii Approach. IMA J. Math. Control Inf. 19 (2002) 157-172.

[26] J. Rauch and M. Taylor, Exponential Decay of Solutions to Hyperbolic Equations in Bounded Domains. Indiana University Math. J. 24 (1975).

[27] D.L. Russell, Canonical Forms and Spectral Determination for a Class of Hyperbolic Distributed Parameter Control Systems. J. Math. Anal. Appl. 62 (1978) 186-225.

[28] D.L. Russell, Neutral FDE Canonical Representations of Hyperbolic Systems. J. Int. Eqs. Appl. 3 (1991) $129-166$.

[29] E.D. Sontag, Smooth Stabilization Implies Coprime Factorization. IEEE Trans. Autom. Control 34 (1989) $435-443$.

[30] R. Vazquez, M. Krstic and J.-M. Coron, Backstepping Boundary Stabilization and State Estimation of a $2 \times 2$ Linear Hyperbolic System, in Proc. of 50th Conf. Decision and Control, Orlando (2011).

[31] C.-Z. Xu and G. Sallet, Exponential Stability and Transfer Functions of Processes Governed by Symmetric Hyperbolic Systems. ESAIM: COCV 7 (2002) 421-442. 